\title{
Decentralized Robust Control of Uncertain Markov Jump Parameter Systems via Output Feedback*
}

\author{
$\mathrm{Li} \mathrm{Li}^{\dagger}$ \\ Valery A. Ugrinovskii ${ }^{\ddagger}$ \\ Robert Orsi ${ }^{\S}$ \\ To appear in Automatica, 2007; doi: 10.1016/j.automatica.2007.03.016
}

\begin{abstract}
This paper addresses the problem of decentralized robust stabilization and control for a class of uncertain Markov jump parameter systems. Control is via output feedback and knowledge of the discrete Markov state. It is shown that the existence of a solution to a collection of mode-dependent coupled algebraic Riccati equations and inequalities, which depend on certain additional parameters, is both necessary and sufficient for the existence of a robust decentralized switching controller. A guaranteed upper bound on robust performance is also given. To obtain a controller which satisfies this bound, an optimization problem involving rank constrained linear matrix inequalities is introduced, and a numerical approach for solving this problem is presented. To demonstrate the efficacy of the proposed approach, an example stabilization problem for a power system comprising three generators and one on-load tap changing transformer is considered.
\end{abstract}

\section{Introduction}

Over the past three decades, there has been extensive interest in the analysis and design of decentralized control for large-scale systems made up of spatially interconnected components. Although centralized controllers for such systems can often be designed using standard control design techniques, in general, centralized control algorithms require a higher level of connectivity and higher communication costs compared to decentralized schemes. Hence, much effort has been focused on the application of decentralized control in such distributed systems, see Sandell, Varaya, Athans, \& Safonov (1978); Siljak (1991) and the references therein.

The problem addressed in this paper is that of robust output feedback control design for an interconnected system subject to uncertain disturbances and random changes in its parameters. Random parameter changes may result from random component failures, repairs or shut down, or abrupt changes of the operating point. Many such events can be modeled using a continuous time finite-state Markov chain, which leads to the hybrid description of system dynamics known as a Markov Jump Parameter (MJP) system (Mariton, 1990); such a description will be utilized in the paper. The state of an MJP system is described by continuous range variables and also a random discrete event variable representing the regime of system

\footnotetext{
${ }^{*}$ This work was supported by the Australian Research Council.

†The author is with the School of Information Technology and Electrical Engineering, University of New South Wales at the Australian Defence Force Academy, ADFA, Canberra, ACT 2600, Australia (email: lleely@itee.adfa.edu.au).

$¥$ The author is with the School of Information Technology and Electrical Engineering, University of New South Wales at the Australian Defence Force Academy, ADFA, Canberra, ACT 2600, Australia (email: v.ougrinovski@adfa.edu.au). Part of this work was done during the author's visit to the Research School of Information Sciences and Engineering, The Australian National University.

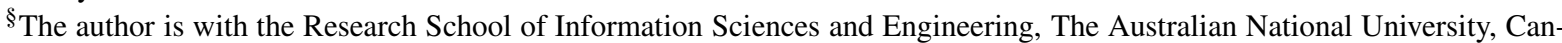
berra, ACT, 0200 Australia (email: robert.orsi@anu.edu.au).
} 
operation. In this paper we assume that the latter variable is independent of continuous dynamics and is available for measurement. There are numerous applications in which such an assumption is valid. For instance, a number of power systems control problems fall in this category (Willsky \& Levy, 1979); below we present two such example problems to illustrate this issue. Mariton (1990) discusses several other applications in which the state of the underlying jump process can be observed. This perfect observation assumption is crucial from the analysis viewpoint (Ji \& Chizeck, 1992), and allows one to avoid much more difficult 'dual control' problems (Caines \& Chen, 1985; Griffiths \& Loparo, 1985). In practice the resulting controller must be implemented together with sensors measuring mode conditions.

Example 1. Power system control under varying load. In power systems, the selection of generator set-points is based on off-line analysis including load forecasting. Currents and voltages at generator buses are constantly monitored, and the load level is computed on a routine basis. On-line sensors then identify a particular set-point for each unit and choose an appropriate controller for that set-point (Qiu, Vittal, \& Khammash, 2004). Following Willsky \& Levy (1979), dynamics of load profile switching can be modeled using a Markov chain; see Ugrinovskii \& Pota (2005). On the other hand, dynamics of the generators are described by differential equations involving generator rotor angles and frequencies. While the frequency is constantly monitored, being a crucial variable, it is usually difficult to measure generator rotor angles directly. Practical difficulties of direct state measurement necessitate the use of output feedback control to regulate transients caused by changing load demands. In Section 7 we give an example to illustrate this problem.

Example 2. Power system control under transmission line tripping and reclosing. Events such as faults in power transmission tie-lines are also closely monitored. Power systems usually operate within safety limits which prevent overloads, however transmission faults may occur as a result of wear, or may be caused by environmental factors. The event sequence starts with the system operating under normal conditions (mode 1). A fault occurring at a random time instant in one of the tie-lines causes protective relays to disconnect the faulty circuit, and the system switches to mode 2. After the fault is cleared, the circuit is reconnected and the system returns to mode 1. In this example, mode switching is driven by random tie-line failures and does not directly depend on the underlying continuous variables of the generators. The mode information is obtained by monitoring the voltage and current at the buses and from the protective relay settings, and can be used in the switching control design (Ramos, Li, Ugrinovskii, \& Pota, 2006).

Robust control of continuous-time systems with random parameter changes modeled as MJPs has attracted much attention in the past decade; see de Souza \& Fragoso (1993); Pan \& Başar (1995); de Farias, Geromel, do Val, \& Costa (2000) and the references therein. Problems of robust decentralized stabilization of uncertain MJP systems were also studied, e.g., see Pakshin (2003). This paper focuses on the problem of absolute decentralized stabilization and robust control of large-scale MJP systems via output feedback. Similar to Ugrinovskii \& Pota (2005), we employ time-domain Integral Quadratic Constraints (IQCs, Yakubovich (1988)) to describe the size of internal perturbations within each subsystem of the large-scale system. Interactions between subsystems are also treated as uncertain perturbations; these interactions are also described in terms of IQCs. We refer to Ugrinovskii, Petersen, Savkin, \& Ugrinovskaya (2000); Ugrinovskii \& Pota (2005) where the IQC uncertainty description was discussed in the context of largescale systems and MJP systems, respectively. Importantly, this uncertainty description allowed to establish an equivalence between the robust control problem considered in those papers and a parameterized family of $H_{\infty}$ control problems for individual subsystems of a system. A special lossless extension of the $S$ procedure suitable for the analysis of MJP systems was derived in Ugrinovskii \& Pota (2005) to establish that result and will also be used in deriving the results of this paper.

This paper considers uncertain interconnected systems and presents a necessary and sufficient condition for the existence of a robust decentralized switching output feedback controller, extending the state feedback design proposed in Ugrinovskii \& Pota (2005). This result involves a collection of mode-dependent 
coupled algebraic Riccati equations and inequalities which depend on certain additional parameters and are related to those for the corresponding $H_{\infty}$ control problem (Pan \& Başar, 1995). An important difference arising from the output feedback formulation is that, using our method, only a suboptimal upper bound on the worst-case output feedback performance can be guaranteed while the state feedback control design proposed in Ugrinovskii \& Pota (2005) is minimax optimal given an initial condition. Our result reduces the design of a suboptimal guaranteed cost controller to solving an optimization problem involving rank constrained linear matrix inequalities (rank constrained LMIs). This enables the possibility of solving the problem numerically. In the full state information case, the rank constraints do not arise, and the problem can be shown to be reducible to a convex optimization problem.

Rank constrained LMIs are an important generalization of standard LMIs. Many control problems that cannot be formulated in the standard LMI framework can be formulated as problems involving rank constrained LMIs, see Orsi, Helmke, \& Moore (2006) and the references therein. Problems involving rank constrained LMIs are in general nonconvex and there currently does not exist a polynomial time algorithm that is able to correctly solve all instances of such problems. Despite this, some of the currently available algorithms can perform well in practice even though they do not guarantee that a solution can always be found. For the example problem discussed in Section 7, the recently developed rank constrained LMI solver LMIRank (Orsi, 2005) is used with good results. Our rank constrained LMI optimization problem arises from a full order dynamic output feedback control problem, and the rank constraints are imposed on auxiliary Lagrange multipliers which parameterize the associated family of $H_{\infty}$ control problems. If the multipliers are held constant, then our problem is convex in the remaining variables. Similar rank constraints on scaling parameters also arise in the scaled $\mu$-synthesis problem (El Ghaoui \& Gahinet, 1993). In contrast, many rank constrained LMI problems are associated with reduced order control design (e.g., see El Ghaoui \& Gahinet (1993)), and the rank constraints are imposed on Lyapunov variables.

The organization of this paper is as follows. In Section 2 we describe the system model and introduce admissible uncertainties. Section 3 shows the equivalence of the robust control problem under consideration and the solvability of the parameterized family of Riccati equations and inequalities. Also in Section 3 we give a performance bound associated with the proposed decentralized control scheme. The proofs of these results are given in Section 4. Next, the problem of optimizing the performance bound is formulated as an optimization problem involving rank constrained LMIs, and a numerical approach for solving this problem is presented in Section 5. In Section 6, the results of Section 3 are extended to guarantee robust asymptotic stability for every mode. We illustrate the method by an example presented in Section 7.

The following notation will be used throughout this paper. $\mathbf{R}^{m}, \mathbf{R}^{m \times n}$ denote the sets of real $m \times 1$ vectors and $m \times n$ matrices. The transpose of a real matrix $M$ is denoted as $M^{\prime}$. For $y \in \mathbf{R}^{m}$ and $M \in$ $\mathbf{R}^{m \times m},\|y\|^{2}=y^{\prime} y$, and $\operatorname{tr}(M)$ is the trace of $M$. We use $\operatorname{diag}\left\{M_{i}\right\}_{i=1}^{N}=\operatorname{diag}\left[M_{1}, \cdots, M_{N}\right]$ to denote a block diagonal matrix with matrices $M_{1}, \cdots, M_{N}$ on its main diagonal, and $\left\{\tau_{i}, \theta_{i}\right\}_{i=1}^{N}$ to denote the vector $\left(\tau_{1}, \theta_{1}, \cdots, \tau_{N}, \theta_{N}\right)^{\prime} \in \mathbf{R}^{2 N} . \mathcal{E}, \mathcal{E}[\cdot \mid \cdot]$ stand for expectation and conditional expectation with respect to the underlying probability measure. Given a continuous time finite state Markov process $\eta(t), t \geq 0$, and its natural filtration, we let $\mathbf{L}_{2}[0, \infty)$ denote the Lebesgue space of square integrable random processes adapted to this filtration; this space is equipped with the norm $\|y(\cdot)\|_{2}^{2}=\mathcal{E} \int_{0}^{+\infty}\|y(t)\|^{2} d t$. 


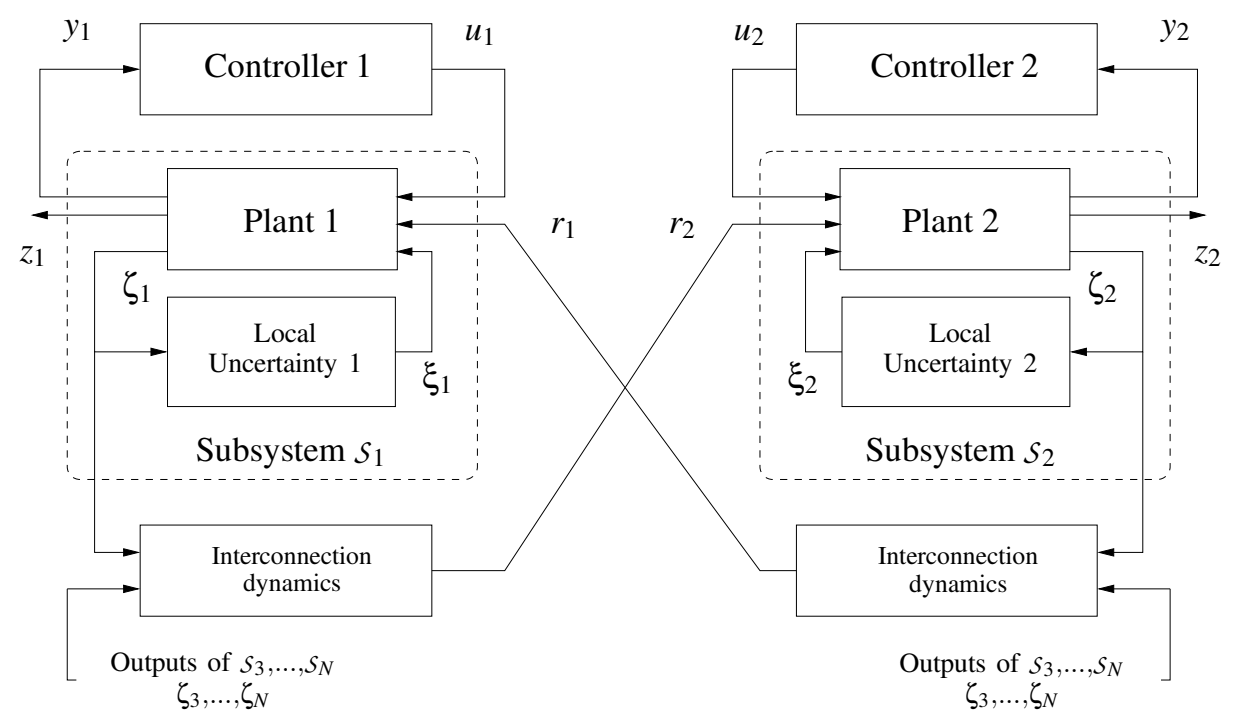

Figure 1: The large-scale uncertain system $\mathcal{S}$. Only two of the $N$ subsystems are shown.

\section{Problem Formulation}

\subsection{System model}

We consider a large-scale system $\mathcal{S}$ comprising $N$ subsystems $S_{i}$ of the following form:

$$
S_{i}:\left\{\begin{array}{c}
\dot{x}_{i}=A_{i}(\eta(t)) x_{i}(t)+B_{i}(\eta(t)) u_{i}(t) \\
+E_{i}(\eta(t)) \xi_{i}(t)+L_{i}(\eta(t)) r_{i}(t), \\
z_{i}=C_{i}(\eta(t)) x_{i}(t)+D_{i}(\eta(t)) u_{i}(t) \\
\zeta_{i}=H_{i}(\eta(t)) x_{i}(t)+G_{i}(\eta(t)) u_{i}(t), \\
y_{i}=\Sigma_{i}(\eta(t)) x_{i}(t)+\Gamma_{i}(\eta(t)) \xi_{i}(t),
\end{array}\right.
$$

where $x_{i} \in \mathbf{R}^{n_{i}}$ is the state, $u_{i} \in \mathbf{R}^{m_{i}}$ is the control input, $\xi_{i} \in \mathbf{R}^{p_{i}}$ is the perturbation, $\zeta_{i} \in \mathbf{R}^{h_{i}}$ is the uncertainty output, $z_{i} \in \mathbf{R}^{q_{i}}$ is the controlled output, $y_{i} \in \mathbf{R}^{g_{i}}$ is the measured output, the input $r_{i}$ describes the effect of the other subsystems $\mathcal{S}_{1}, \ldots, \mathcal{S}_{i-1}, \mathcal{S}_{i+1}, \ldots, \mathcal{S}_{N}$ on subsystem $\mathcal{S}_{i}$, and $\eta(t)$ describes the mechanism of mode switching. The structure of the system $S$ is shown in Figure 1.

It is assumed that $\eta(t)$ is a homogeneous stationary Markov chain defined on a complete probability space $(\Omega, \mathcal{F}, \mathcal{P})$ and taking values in a finite set $\mathbb{K}=\{1,2, \cdots, k\}$. Its state transition probability matrix $\mathbf{P}(t)=[\mathcal{P}\{\boldsymbol{\eta}(t+s)=\mu \mid \eta(s)=v\}]_{\mathrm{v}, \mu=1}^{k}$ is independent of $s \geq 0$ and under mild conditions is known to have the form $\mathbf{P}(t)=e^{\mathbf{Q} t}$, where $\mathbf{Q} \triangleq\left[q_{v \mu}\right]_{v, \mu=1}^{k}$ is a matrix in which $q_{v \mu} \geq 0, v \neq \mu$, and $q_{v v}=-\sum_{\mu \neq v} q_{v \mu}$. Furthermore, this process will be assumed to have properties that warrant the existence of a semigroup of measure-preserving one-to-one point or set transformations $\Upsilon_{t}: \Omega \rightarrow \Omega$ such that for all $t, s \geq 0, \eta(t+$ $s, \omega)=\eta\left(t, \Upsilon_{s} \omega\right)$ a.s. (Doob, 1953). The stationary initial distribution $\pi=\left[\pi_{1}, \cdots, \pi_{k}\right]$ of the process $\eta(t)$ will be assumed to be positive, i.e., $\pi_{j}>0, \forall j \in \mathbb{K}$.

The assumptions which ensure the above mentioned structure of the state-transition matrix $\mathbf{P}$ are standard in the theory of MJP systems; e.g., see Mariton (1990). The additional technical assumption of the existence of a semigroup of measure-preserving transformations will allow us to employ the stochastic $S$-procedure result of Ugrinovskii \& Pota (2005). Such a semigroup exists if the process $\eta(t)$ is strictly stationary, and $\pi_{j}>0, \forall j \in \mathbb{K}$, as assumed above (Doob, 1953). Note that the assumption about the existence of such a semigroup is used only in the proof of the necessity part of Theorem 12. For the 'if' claim to be true, weaker properties of $\eta(t)$ may be sufficient, for instance, $\pi_{j} \geq 0, \forall j \in \mathbb{K}$. 
In practical applications, systems of the form (1) arise from linearizing a nonlinear plant about several operating points. The resulting model allows for handling small disturbances caused by perturbations about operating points via local controllers, while large disturbances caused by the system transitions from one operating point to another are regarded as mode changes and are handled by controller switching.

The derivation of the proposed robust control algorithm requires a number of standard technical assumptions about the system $\mathcal{S}$. These assumptions will allow us to use the existing results on stochastic $H_{\infty}$ control of MJP systems in our problem; in fact, some of the assumptions presented below are due to Pan \& Başar (1995). Assumption 3 is a basic assumption stating that the system $\mathcal{S}$ is well defined on each finite time interval $[0, T]$. Assumptions 4-6 state basic controllability and observability properties of the uncertain system $\mathcal{S}$; they will be used to prove the results involving coupled Riccati equations and inequalities; see Theorem 12. Such assumptions are often used in the Riccati approach to robust control design, cf. Başar \& Bernhard (1995); they allow one to infer from the robust stabilizability of the system that the corresponding Riccati equations have stabilizing solutions. The assumptions below must hold for each subsystem $\mathcal{S}_{i}, i=1, \cdots, N$, and each state $j \in \mathbb{K}$ of the process $\eta(t)$.

Assumption 3. Given any locally square integrable triple of signals $\left(u_{i}(\cdot), r_{i}(\cdot), \xi_{i}(\cdot)\right)$, for any initial condition $x_{i}(0)=x_{i 0}, \eta(0)=\eta_{0}$, the solution to each subsystem equation exists on any finite time interval $[0, T]$ and is locally square integrable; i.e. $\mathcal{E}\left[\int_{0}^{T}\left\|x_{i}(\cdot)\right\|^{2} d t \mid x_{i 0}, \eta_{0}\right]<\infty$.

Assumption 4. $D_{i}^{\prime}(j) D_{i}(j)+G_{i}^{\prime}(j) G_{i}(j)>0, \Gamma_{i}(j) \Gamma_{i}^{\prime}(j)>0$.

Assumption 5. Each pair $\left(A_{i}(j), C_{i}^{\prime}(j) C_{i}(j)\right)$ is observable.

Assumption 6. Each subsystem $\mathcal{S}_{i}$ is stochastically stabilizable via input $u_{i}(c f$. Ji \& Chizeck (1990)). That $i$, there exists a linear state-feedback control law $u=\bar{K}_{i}(\eta(t)) x$ and a symmetric positive definite matrix $\bar{M}_{i}$ such that solutions of the system

$$
\dot{x}_{i}=\left[A_{i}(\eta(t))+B_{i}(\eta(t)) \bar{K}_{i}(\eta(t))\right] x_{i}(t)
$$

with $x_{i}(0)=x_{i 0}, \eta(0)=\eta_{0}$ satisfy the condition

$$
\lim _{T \rightarrow \infty} \mathcal{E}\left[\int_{0}^{T}\left\|x_{i}(t)\right\|^{2} d t \mid x_{i 0}, \eta_{0}\right] \leq x_{i 0}^{\prime} \bar{M}_{i} x_{i 0}
$$

Remark 7. The notion of stochastic stabilizability is equivalent to that of mean square (MS) stabilizability which requires that for all $x_{i 0}$ and $\eta_{0}, \mathcal{E}\left[\left\|x_{i}(t)\right\|^{2} \mid x_{i 0}, \eta_{0}\right] \rightarrow 0$ as $t \rightarrow \infty$ (Feng, Loparo, Ji, \& Chizeck, 1992). Also, since $\pi_{j}>0$ for all $j \in \mathbb{K}$, (2) is equivalent to the condition $\|x(\cdot)\|_{2}^{2} \leq x_{i 0}^{\prime} \bar{M}_{i} x_{i 0} \forall x_{i 0}$. An LMI condition to check Assumption 6 can be found in Ait Rami \& El Ghaoui (1996).

In addition to these assumptions, it will be convenient to assume that $C_{i}^{\prime}(j) D_{i}(j)=0, H_{i}^{\prime}(j) G_{i}(j)=0$, $E_{i}(j) \Gamma_{i}^{\prime}(j)=0$. It is well known that these conditions can be easily met using an appropriate transformation of variables (Başar \& Bernhard, 1995). Also, we will assume that $\left[E_{i}(j) L_{i}(j)\right] \neq 0$. This means that the system $\mathcal{S}$ does not contain perfectly known subsystems which are disconnected from the rest of the system. This assumption is not restrictive, since if such a subsystem exists, it can be dealt with independently.

\subsection{Feasible uncertainties}

We now present mathematical descriptions of the feasible uncertain perturbations for the large-scale system $\mathcal{S}$. In each subsystem $\mathcal{S}_{i}$, see (1), the signal $\xi_{i}(\cdot)$ accounts for the presence of uncertain dynamics which are driven by the uncertainty output $\zeta_{i}(\cdot)$ generated within the subsystem; see Figure 1. Hence we refer to $\xi_{i}(\cdot)$ as the local uncertainty for the subsystem $S_{i}$. Also each subsystem $S_{i}$ is driven by the interconnection signal $r_{i}(\cdot)$. Since each $r_{i}(\cdot)$ is generated by uncertain subsystems other than $S_{i}$, the interconnections 
can also be regarded as uncertain perturbations acting on $S_{i}$ whose effect is additional to that of local disturbances. We refer to the $r_{i}(\cdot)$ 's as interconnection uncertainties. Treating system interconnections as uncertainties has been quite common (Siljak, 1991). This approach presumes that for the derivation of a decentralized controller, the unperturbed nominal design system can be partitioned into uncoupled subsystems, and interconnections present in the true system are accounted for through the requirement that the controller must provide a sufficient 'interconnection attenuation' for the overall closed-loop system to retain stability and acceptable performance. The introduction of the jump variable provides an extra 'degree of freedom' to allow one to consider systems in which unperturbed regimes vary.

As mentioned we assume that both local uncertainties and interconnections of the large-scale system $\mathcal{S}$ are represented by input signals satisfying certain IQCs. The definitions below follow Ugrinovskii \& Pota (2005). They employ two sets of symmetric matrices, $M_{i}>0, \hat{M}_{i}>0, i=1, \cdots, N$.

Definition 8. A collection of uncertainty inputs $\xi_{i}(\cdot), i=1, \cdots, N$, represents an admissible uncertainty for the large-scale system $S$ if, given any locally square integrable control inputs $u_{i}(\cdot)$ and locally square integrable interconnection inputs $r_{i}(\cdot), i=1, \cdots, N$, there exists a sequence $\left\{t_{l}\right\}_{l=1}^{+\infty}, t_{l} \rightarrow+\infty$, such that for all $i=1, \cdots, N$,

$$
\mathcal{E} \int_{0}^{t_{l}}\left(\left\|\zeta_{i}(t)\right\|^{2}-\left\|\xi_{i}(t)\right\|^{2}\right) d t \geq-x_{i 0}^{\prime} M_{i} x_{i 0} .
$$

The set of all such admissible uncertainties is denoted by $\Xi$.

Definition 9. The large-scale system $\mathcal{S}$ is said to have admissible interconnections if, given any locally square integrable control inputs $u_{i}(\cdot)$ and locally square integrable uncertainty inputs $\xi_{i}(\cdot), i=1, \cdots, N$, there exists a sequence $\left\{t_{l}\right\}_{l=1}^{+\infty}, t_{l} \rightarrow+\infty$, such that

$$
\mathcal{E} \int_{0}^{t_{l}}\left(\sum_{n=1, n \neq i}^{N}\left\|\zeta_{n}(t)\right\|^{2}-\left\|r_{i}(t)\right\|^{2}\right) d t \geq-x_{i 0}^{\prime} \hat{M}_{i} x_{i 0}
$$

for all $i=1, \cdots, N$. The corresponding collection of interconnection inputs $r_{i}(\cdot), i=1, \cdots, N$, is called an admissible interconnection, and the set of such admissible interconnections is denoted by $\Pi$.

Without loss of generality, we assume the same sequences of $\left\{t_{l}\right\}_{l=1}^{+\infty}$ in Definitions 8 and 9 whenever they correspond to the same collection of signals $\xi_{i}(\cdot), r_{i}(\cdot), \zeta_{i}(\cdot)$. These definitions capture a broad class of uncertainties arising from unknown nonlinearities or unmodeled dynamics (Petersen, Ugrinovskii, \& Savkin, 2000). They also allow to account for effects of non-zero initial conditions of uncertain dynamics as well as noises and delays in the local uncertainty channels and interconnections. The terms on the right hand sides of the IQCs (3) and (4) correspond to bounds on these effects. We follow Savkin \& Petersen (1995) and write those bounds as quadratic forms $x_{i 0}^{\prime} M_{i} x_{i 0}, x_{i 0}^{\prime} \hat{M}_{i} x_{i 0}$; more generally, these bounds can be expressed as some constants (Petersen et al., 2000). Allowing for a wider class of uncertainties which satisfy the IQCs makes it possible to apply the $S$-procedure for MJP systems (Ugrinovskii \& Pota, 2005) to establish the equivalence between our robust control problem and a set of parameterized $H_{\infty}$ control problems for each subsystem $\mathcal{S}_{i}$. As illustrated below, the norm bounded uncertainty is a special case of the IQC uncertainty model.

Example 10. (cf. Petersen \& McFarlane (1994); Wang, Xie, \& de Souza (1995).) Consider the deterministic uncertain system consisting of $N$ subsystems

$$
\dot{x}_{i}=\left(A_{i}+\Delta A_{i}\right) x_{i}+\left(B_{i}+\Delta B_{i}\right) u_{i}+\sum_{j \neq i}\left(A_{i j} x_{j}+B_{i j} u_{j}\right)
$$

the parametric uncertainties $\Delta A_{i}, \Delta B_{i}$ and the interconnections are defined using some known matrices $E_{i}, L_{i}, H_{i}, G_{i}$ and uncertain parameter matrices $\Delta_{i}(t), \hat{\Delta}_{i j}(t)$ as follows

$$
\begin{array}{r}
{\left[\Delta A_{i} \Delta B_{i}\right]=E_{i} \Delta_{i}(t)\left[H_{i} G_{i}\right], \quad\left[A_{i j} B_{i j}\right]=L_{i} \hat{\Delta}_{i j}(t)\left[H_{j} G_{j}\right],} \\
\Delta_{i}^{\prime}(t) \Delta_{i}(t) \leq I, \quad \sum_{j \neq i} \hat{\Delta}_{i j}(t) \hat{\Delta}_{i j}^{\prime}(t) \leq I .
\end{array}
$$


Then, by defining the interconnection inputs and outputs as

$$
\zeta_{i}=H_{i} x_{i}+G_{i} u_{i}, \quad \xi_{i}=\Delta_{i}(t) \zeta_{i}, \quad r_{i}=\sum_{j \neq i} \hat{\Delta}_{i j}(t) \zeta_{j}
$$

each subsystem (5) can be written as a single-mode subsystem $S_{i}$ of the form (1) subject to the constraints

$$
\left\|\xi_{i}(t)\right\|^{2} \leq\left\|\zeta_{i}(t)\right\|^{2}, \quad\left\|r_{i}(t)\right\|^{2} \leq \sum_{j \neq i}\left\|\zeta_{j}(t)\right\|^{2} .
$$

The IQCs (3) and (4) are then satisfied with any $M_{i}, \hat{M}_{i}>0$.

\subsection{Absolute stabilization via decentralized control}

For the uncertain large-scale system $\mathcal{S}$ comprising subsystems $S_{i}$ of the form (1) and subject to the constraints (3) and (4), we consider the following problem of absolute stabilization by means of decentralized linear output feedback controllers of the form

$$
\begin{aligned}
\dot{x}_{c, i}(t) & =A_{c, i}(\eta(t)) x_{c, i}(t)+B_{c, i}(\eta(t)) y_{i}(t), \\
u_{i}(t) & =K_{c, i}(\eta(t)) x_{c, i}(t),
\end{aligned}
$$

where $x_{c, i} \in \mathbf{R}^{n_{c, i}}$ is the $i$ th controller state vector.

Definition 11. The MJP large-scale system $S$ subject to perturbations and interconnections satisfying the constraints (3) and (4) is said to be absolutely stabilizable via decentralized output feedback control if there exists a collection of controllers of the form (6) and a constant $c_{1}>0$ such that for any initial conditions $\left[x_{i}^{\prime}(0), x_{c, i}^{\prime}(0)\right]^{\prime}$, any admissible local uncertainty inputs $\xi_{i}(\cdot)$ and any admissible interconnection inputs $r_{i}(\cdot)$, the signals $x_{i}, x_{c, i}, u_{i}, \xi_{i}, r_{i}$ belong to $L_{2}[0,+\infty)$ and are uniformly bounded,

$$
\begin{gathered}
\sum_{i=1}^{N}\left(\left\|x_{i}(\cdot)\right\|_{2}^{2}+\left\|x_{c, i}(\cdot)\right\|_{2}^{2}+\left\|u_{i}(\cdot)\right\|_{2}^{2}+\left\|\xi_{i}(\cdot)\right\|_{2}^{2}+\left\|r_{i}(\cdot)\right\|_{2}^{2}\right) \\
\leq c_{1} \sum_{i=1}^{N}\left(\left\|x_{i}(0)\right\|^{2}+\left\|x_{c, i}(0)\right\|^{2}\right) .
\end{gathered}
$$

The stability property (7) provides a bound on the norm of closed-loop system transients, which is uniform with respect to admissible uncertainties and interconnections. This justifies referring to it as an absolute stability property.

One approach to achieve robust stabilization is to seek a controller of the form (6) which achieves a bounded system performance measured in terms of the 'worst-case' quadratic performance cost

$$
J_{w c}(u) \triangleq \sup _{\Xi, \Pi} \sum_{i=1}^{N}\left\|z_{i}\right\|_{2}^{2}
$$

where $\Xi$ and $\Pi$ are the sets defined in Definitions 8 and 9. It is often possible to show that this controller is also absolutely stabilizing in the sense of Definition 11; we refer to Petersen et al. (2000) for extensive coverage on this approach to robust stabilization. We note that in some problems, given a set $u$ of allowable controllers, it is possible to find a controller which attains an optimal worst-case system performance $\inf _{u} J_{w c}(u)$. For other problems, such as the one considered in this paper, it is only possible to obtain a controller of the form (6) guaranteeing a bound on $\inf _{u} J_{w c}(u)$. In the next section, we present a necessary and sufficient condition for the existence of an absolutely stabilizing controller and discuss robust performance of such controllers. 


\section{Necessary and sufficient conditions for absolute stabilizability and a sub- optimal control design}

Let $\tau_{i}>0, \theta_{i}>0, i=1, \cdots, N$, be given constants, and $\bar{\theta}_{i}=\sum_{n=1, n \neq i}^{N} \theta_{n}$. We consider a collection of coupled generalized algebraic Riccati equations (GAREs) and generalized algebraic Riccati inequalities (GARIs) for $j=1, \cdots, k$ :

$$
\begin{aligned}
& A_{i}^{\prime}(j) X_{i}(j)+X_{i}(j) A_{i}(j)+\sum_{v=1}^{k} q_{j v} X_{i}(v)+\bar{C}_{i}^{\prime}(j) \bar{C}_{i}(j) \\
& -X_{i}(j)\left[B_{i}(j) R_{i}^{-1}(j) B_{i}^{\prime}(j)-\bar{B}_{2, i}(j) \bar{B}_{2, i}^{\prime}(j)\right] X_{i}(j)=0 \\
& A_{i}^{\prime}(j) Y_{i}(j)+Y_{i}(j) A_{i}(j)+Y_{i}(j) \bar{B}_{2, i}(j) \bar{B}_{2, i}^{\prime}(j) Y_{i}(j) \\
& -\left[\Sigma_{i}^{\prime}(j) W_{i}^{-1}(j) \Sigma_{i}(j)-\bar{C}_{i}^{\prime}(j) \bar{C}_{i}(j)\right]+\sum_{v=1}^{k} q_{j v} Y_{i}(v)<0
\end{aligned}
$$

where $R_{i}(j)=\bar{D}_{i}^{\prime}(j) \bar{D}_{i}(j), W_{i}(j)=\bar{\Gamma}_{i}(j) \bar{\Gamma}_{i}^{\prime}(j)$ are positive definite matrices, as guaranteed by Assumption 4 , and

$$
\begin{gathered}
\bar{C}_{i}(j)=\left[\begin{array}{c}
C_{i}(j) \\
\sqrt{\tau_{i}+\bar{\theta}_{i}} H_{i}(j)
\end{array}\right], \bar{D}_{i}(j)=\left[\begin{array}{c}
D_{i}(j) \\
\sqrt{\tau_{i}+\bar{\theta}_{i}} G_{i}(j)
\end{array}\right], \\
\bar{B}_{2, i}(j)=\left[\tau_{i}^{-\frac{1}{2}} E_{i}(j), \theta_{i}^{-\frac{1}{2}} L_{i}(j)\right], \bar{\Gamma}_{i}(j)=\left[\tau_{i}^{-\frac{1}{2}} \Gamma_{i}(j), 0\right] .
\end{gathered}
$$

Associated with (9) and (10) is a collection of decentralized dynamic output feedback controllers of the form (6) in which

$$
\begin{aligned}
K_{c, i}(j)= & -R_{i}^{-1}(j) B_{i}^{\prime}(j) X_{i}(j) \\
B_{c, i}(j)= & {\left[Y_{i}(j)-X_{i}(j)\right]^{-1} \Sigma_{i}^{\prime}(j) W_{i}^{-1}(j), } \\
A_{c, i}(j)= & A_{i}(j)-B_{c, i}(j) \Sigma_{i}(j)+B_{i}(j) K_{c, i}(j) \\
& +\bar{B}_{2, i}(j) \bar{B}_{2, i}^{\prime}(j) X_{i}(j) .
\end{aligned}
$$

The following set will play an important role in the control design; see Theorems 12 and 13 given below:

$$
\begin{aligned}
\mathcal{T}= & \left\{\left\{\tau_{i}, \theta_{i}\right\}_{i=1}^{N} \in \mathbf{R}^{2 N}, \tau_{i}>0, \theta_{i}>0:\right. \text { the GAREs (9) } \\
& \text { admit minimal positive definite solutions } X_{i}(j), j \in \mathbb{K}, \\
& \text { and the GARIs }(10) \text { admit solutions } Y_{i}(j)>0, j \in \mathbb{K}, \\
& \text { such that } \left.Y_{i}(j)>X_{i}(j) \forall j \in \mathbb{K}\right\} .
\end{aligned}
$$

Note that the matrices $X_{i}(j), Y_{i}(j)$ defined above depend on the chosen $\left\{\tau_{i}, \theta_{i}\right\}_{i=1}^{N} \in \mathcal{T}$. This dependence is assumed throughout the paper. It allows us to define, using the solutions of the GAREs (9), the following function on the set $\mathcal{T}$ :

$$
\begin{aligned}
J(\tau, \theta) & \triangleq \sum_{i=1}^{N} x_{i 0}^{\prime} \Psi_{i}\left(X_{i}, \tau_{i}, \theta_{i}\right) x_{i 0} \\
\Psi_{i}\left(Z_{i}, \tau_{i}, \theta_{i}\right) & \triangleq \sum_{j=1}^{k} \pi_{j} Z_{i}(j)+\tau_{i} M_{i}+\theta_{i} \hat{M}_{i}, \quad Z_{i}(j) \in \mathbf{R}^{n_{i} \times n_{i}} .
\end{aligned}
$$

Now we are in a position to state the main results of this section. Theorem 12 presents a necessary and sufficient condition for the uncertain interconnected system $\mathcal{S}$ to be absolutely stabilizable by a decentralized controller (6). Theorem 13 characterizes guaranteed robust performance achievable by such a controller. Proofs of these theorems are given in the next section. 
Theorem 12. Consider the large-scale system $S$ in which the uncertainties and interconnections satisfy the constraints (3) and (4). This system is absolutely stabilizable via a decentralized dynamic output feedback control of the form (6) if and only if the set $\mathcal{T}$ is non-empty.

Theorem 13. Given a vector of initial conditions $x_{i}(0)=x_{i 0}$, consider the set $\mathcal{u}$ of decentralized controllers (6). Suppose the set $\mathcal{T}$ is not empty and $\left\{\tau_{i}^{*}, \theta_{i}^{*}\right\}_{i=1}^{N} \in \mathcal{T}$ attains the infimum $\inf _{\mathcal{T}} J(\tau, \theta)$. Then a decentralized controller $u^{*}$ whose worst-case performance (8) satisfies the upper bound

$$
J_{w c}\left(u^{*}\right) \leq J\left(\tau^{*}, \theta^{*}\right)=\inf _{\mathcal{T}} J(\tau, \theta)
$$

is given by (6), (12) in which $\tau_{i}=\tau_{i}^{*}, \theta_{i}=\theta_{i}^{*}, i=1, \cdots, N$, with the initial condition $x_{c, i}(0)=x_{i}(0)$. Consequently, the optimal worst-case closed-loop system performance achievable via decentralized controllers of the class $\mathcal{u}$ is upper-bounded as follows,

$$
\inf _{\mathcal{u}} J_{w c}(u) \leq \inf _{\mathcal{T}} J(\tau, \theta) .
$$

To achieve the claimed bound on optimal worst-case performance, the optimization problem defined on the right-hand side of (15) must be solved, which requires knowledge of the plant initial states $x_{i}(0)$. In practice, however, the initial state of the plant may not be known. It is possible to avoid using $x_{i}(0)$ in this optimization problem. One possibility is to assume that $x_{i}(0)$ is a zero mean Gaussian random variable with unity covariance, and to minimize the expectation of $J(\tau, \theta)$ taken with respect to this random variable, which is given by $\sum_{i=1}^{N} \operatorname{tr}\left[\Psi_{i}\left(X_{i}, \tau_{i}, \theta_{i}\right)\right]$; cf. Petersen \& McFarlane (1994). Another idea is to minimize the sum of the largest eigenvalues of $\Psi_{i}\left(X_{i}, \tau_{i}, \theta_{i}\right)$, or a scaled sum thereof. These approaches will lead to suboptimal guaranteed cost controllers independent of the precise values of $x_{i}(0)$.

The controller in Theorem 13 is initialized by setting $x_{c, i}(0)=x_{i}(0)$. This also can be avoided. As will be seen from the proof of this theorem, when $x_{i}(0)$ are not known we can let $x_{c, i}(0)=0$. This will yield a controller with a higher bound on the system performance given by

$$
\inf _{\mathcal{T}} \sum_{i=1}^{N} x_{i 0}^{\prime} \Psi_{i}\left(Y_{i}, \tau_{i}, \theta_{i}\right) x_{i 0}
$$

We conclude the section by noting that in the state feedback case (Ugrinovskii \& Pota, 2005), the corresponding controller $u^{*}$ was shown to be minimax optimal, i.e., (14) and (15) were exact identities. In the output feedback case, Theorem 13 only guarantees a suboptimal upper bound on the worst-case performance.

\section{Proofs of Theorems 12 and 13}

The proofs presented in this section use a novel technique based on the results of Li \& Ugrinovskii (2006).

\subsection{Proof of the 'only if' part of Theorem 12: the necessary condition for absolute stabi- lizability}

Suppose the given uncertain MJP large-scale system $\mathcal{S}$ is absolutely stabilizable via decentralized output feedback control. Then we conclude from (7) that there exists a decentralized controller of the form (6) and a finite constant $c>0$ such that

$$
J_{w c}(u)<c .
$$

We wish to infer from this condition that the set $\mathcal{T}$ is not empty. The proof of this statement will proceed as follows. First, using the S-procedure result of Ugrinovskii \& Pota (2005), we will show that the condition 
(16) implies the solvability of a certain family of scaled jump-parameter $H_{\infty}$ control problems. Each problem is formulated for a subsystem of an uncertain system corresponding to a scaled version of the system (1), and involves some scaling parameters $\tau_{i}, \theta_{i}$. The next step will be to use the existing results on the $H_{\infty}$ control of MJP systems to establish that the vector of scaling parameters $\left\{\tau_{i}, \theta_{i}\right\}_{i=1}^{N}$ belongs to the set $\mathcal{T}$.

For an arbitrary pair of elements of $\Xi$ and $\Pi$, let us write the corresponding closed loop system in the form

$$
\dot{\bar{x}}_{i}(t)=\bar{A}_{i}(\eta(t)) \bar{x}_{i}(t)+\tilde{B}_{2, i}(\eta(t)) w_{i}(t)
$$

where

$$
\begin{aligned}
& \bar{A}_{i}(j)=\left[\begin{array}{cc}
A_{i}(j) & B_{i}(j) K_{c, i}(j) \\
B_{c, i}(j) \Sigma_{i}(j) & A_{c, i}(j)
\end{array}\right], \quad \tilde{B}_{2, i}(j)=\left[\begin{array}{cc}
E_{i}(j) & L_{i}(j) \\
B_{c, i}(j) \Gamma_{i}(j) & 0
\end{array}\right], \\
& \bar{x}_{i}(t)=\left[x_{i}^{\prime}(t) \quad x_{c, i}^{\prime}(t)\right]^{\prime}, \quad w_{i}(t)=\left[\xi_{i}^{\prime}(t) \quad r_{i}^{\prime}(t)\right]^{\prime}, \quad w=\left[w_{1}^{\prime}, \cdots, w_{N}^{\prime}\right]^{\prime} .
\end{aligned}
$$

In view of (16), one can choose a sufficiently small constant $\varepsilon>0$ such that

$$
(1+\varepsilon) \mathcal{E} \int_{0}^{+\infty} \sum_{i=1}^{N}\left\|z_{i}\right\|^{2} d t<c-\varepsilon
$$

Define the quadratic functionals

$$
\begin{aligned}
\mathcal{G}_{0}(w) & =(1+\varepsilon) \mathcal{E} \int_{0}^{+\infty} \sum_{i=1}^{N}\left\|z_{i}\right\|^{2} d t-c+\varepsilon \\
\mathcal{G}_{i, 1}(w) & =\mathcal{E} \int_{0}^{+\infty}\left(\left\|\zeta_{i}(t)\right\|^{2}-\left\|\xi_{i}(t)\right\|^{2}\right) d t+x_{i 0}^{\prime} M_{i} x_{i 0}, \\
\mathcal{G}_{i, 2}(w) & =\mathcal{E} \int_{0}^{+\infty}\left(\sum_{\substack{n=1 \\
n \neq i}}^{N}\left\|\zeta_{n}(t)\right\|^{2}-\left\|r_{i}(t)\right\|^{2}\right) d t+x_{i 0}^{\prime} \hat{M}_{i} x_{i 0} .
\end{aligned}
$$

Now consider a set of inputs $w \in L_{2}[0,+\infty)$ for which

$$
\mathcal{G}_{i, 1}(w) \geq 0, \quad \mathcal{G}_{i, 2}(w) \geq 0 .
$$

Condition (19) implies that each such input satisfies the constraints (3) and (4) with $t_{l}=+\infty$. Therefore, in view of the assumption that the chosen decentralized controller guarantees the satisfaction of condition (16), inequality (19) implies that $\mathcal{G}_{0}(w)<0$. Furthermore, since $M_{i}>0, \hat{M}_{i}>0$, one can choose an input $w$ to satisfy condition (ii) of Lemma 2 of Ugrinovskii \& Pota (2005). According to the S-procedure result given by Lemma 2 of Ugrinovskii \& Pota (2005), these facts imply that one can find constants $\tau_{i} \geq 0$, $\theta_{i} \geq 0, i=1, \cdots, N$, such that

$$
\mathcal{G}_{0}(w)+\sum_{i=1}^{N}\left(\tau_{i} \mathcal{G}_{i, 1}(w)+\theta_{i} \mathcal{G}_{i, 2}(w)\right) \leq 0
$$

for any input $w \in L_{2}[0,+\infty)$. Using the notation $I(w)=\mathcal{E} \int_{0}^{+\infty} \sum_{i=1}^{N}\left\|z_{i}\right\|^{2} d t$, this conclusion can be written as follows:

$$
\begin{aligned}
& I(w)+\mathcal{E} \int_{0}^{+\infty}\left[\left(\sum_{i=1}^{N}\left(\tau_{i}+\bar{\theta}_{i}\right)\left\|\zeta_{i}\right\|^{2}\right)-\|\bar{w}\|^{2}\right] d t \leq-\varepsilon I(w)+c-\varepsilon-\sum_{i=1}^{N} x_{i 0}^{\prime}\left(\tau_{i} M_{i}+\theta_{i} \hat{M}_{i}\right) x_{i 0}, \\
& \bar{w}_{i}=\left[\begin{array}{cc}
\tau_{i}^{1 / 2} \xi_{i}^{\prime}(t) & \theta_{i}^{1 / 2} r_{i}^{\prime}(t)
\end{array}\right]^{\prime}, \quad \bar{w}=\left[\bar{w}_{1}^{\prime}, \cdots, \bar{w}_{N}^{\prime}\right]^{\prime} .
\end{aligned}
$$


Furthermore, it can be shown that condition (20) implies that $\tau_{i}>0, \theta_{i}>0$. The proof of this fact follows the lines proving Proposition 2 and 3 of Section 5.3 of Petersen et al. (2000); also, see the original paper Savkin \& Petersen (1995). We also note that in the case of zero initial conditions, $x_{i}(0)=0, x_{c, i}(0)=0$, (20) implies

$$
I(w)+\mathcal{E} \int_{0}^{+\infty}\left[\left(\sum_{i=1}^{N}\left(\tau_{i}+\bar{\theta}_{i}\right)\left\|\zeta_{i}\right\|^{2}\right)-\|\bar{w}\|^{2}\right] d t \leq-\varepsilon I(w) \quad \forall w \in L_{2}[0,+\infty) ;
$$

see Proposition 2 of Section 5.3 of Petersen et al. (2000) and Savkin \& Petersen (1995). Condition (21) implies that the closed loop augmented MJP system corresponding to the chosen controller (6),

$$
\begin{aligned}
& \dot{\bar{x}}(t)=\bar{A}(\eta(t)) \bar{x}(t)+\bar{B}_{2}(\eta(t)) \bar{w}(t), \\
& \bar{z}(t)=\bar{C}(\eta(t)) \bar{x}(t), \\
& \bar{A}(j)=\operatorname{diag}\left\{\bar{A}_{i}(j)\right\}_{i=1}^{N}, \quad \bar{x}=\left[\bar{x}_{1}^{\prime}, \cdots, \bar{x}_{N}^{\prime}\right]^{\prime}, \\
& \bar{B}_{2}(j)=\operatorname{diag}\left\{\left[\begin{array}{c}
\bar{B}_{2, i}(j) \\
B_{c, i}(j) \bar{\Gamma}_{i}(j)
\end{array}\right]\right\}_{i=1}^{N}, \\
& \bar{C}(j)=\operatorname{diag}\left\{\left[\begin{array}{ll}
\bar{C}_{i}(j) & \left.\left.\bar{D}_{i}(j) K_{c, i}(j)\right]\right\}_{i=1}^{N}, \quad j \in \mathbb{K},
\end{array}\right.\right.
\end{aligned}
$$

satisfies the following $H_{\infty}$-type condition:

$$
\sup _{\substack{\bar{w} \neq 0 \\ \bar{w} \in L_{2}[0,+\infty)}} \frac{\mathcal{E} \int_{0}^{+\infty}\|\bar{z}\|^{2} d t}{\mathcal{E} \int_{0}^{+\infty}\|\bar{w}\|^{2} d t}<1 .
$$

Here, $\bar{z}$ is the output of the system (22) corresponding to the initial condition $\bar{x}(0)=0$ and a random $\eta(0)$ distributed according to the probability distribution $\pi$.

Condition (23) implies that for each $i=1, \cdots, N$,

$$
\left\|\mathbf{T}_{\bar{z}_{i} \bar{w}_{i}}\right\|_{\infty} \triangleq \sup _{\substack{\bar{x}_{i}(0)=0, \bar{w}_{i} \neq 0 \\ \bar{w}_{i} \in L_{2}[0,+\infty)}} \frac{\left\|\bar{z}_{i}(\cdot)\right\|_{2}^{2}}{\left\|\bar{w}_{i}(\cdot)\right\|_{2}^{2}}<1
$$

where $\bar{w}_{i}$ is the disturbance input of the closed loop subsystem consisting of the open loop subsystem

$$
\begin{aligned}
& \dot{x}_{i}(t)=A_{i}(\eta(t)) x_{i}(t)+B_{i}(\eta(t)) u_{i}(t)+\bar{B}_{2, i}(\eta(t)) \bar{w}_{i}(t), \\
& \bar{z}_{i}(t)=\bar{C}_{i}(\eta(t)) x_{i}(t)+\bar{D}_{i}(\eta(t)) u_{i}(t), \\
& y_{i}(t)=\Sigma_{i}(\eta(t)) x_{i}(t)+\bar{\Gamma}_{i}(\eta(t)) \bar{w}_{i}(t),
\end{aligned}
$$

and the $i$-th entry of the considered controller (6); here $\mathbf{T}_{\bar{z}_{i} \bar{w}_{i}}$ is the mapping $\bar{w}_{i} \rightarrow \bar{z}_{i}$ defined by the corresponding closed loop system. To verify this fact, it is sufficient to let $\bar{w}_{j}(\cdot)=0, j \neq i$, in (23) and (22). Indeed, all entries $\bar{z}_{j}(\cdot), j \neq i$, of the corresponding output vector of the system (22) will be equal to zero, hence (24) follows from (23).

Now we observe that the closed-loop subsystem corresponding to (25) and the local controller $u_{i}$ is internally stochastically stable. Indeed, note that $\xi_{i} \equiv 0, r_{i} \equiv 0$ are in the sets of admissible uncertainty inputs and admissible interconnection inputs $\Xi, \Pi$. Letting $\bar{w}_{i} \equiv 0$, by (7), given any $\bar{x}_{i}(0), \eta(0)$, we have

$$
\left\|\bar{x}_{i}(\cdot)\right\|_{2}^{2}<+\infty .
$$

Since $\pi_{j}>0 \forall j \in \mathbb{K}$, this verifies the internal stochastic stability of the closed-loop subsystem for all $\bar{x}_{i}(0)$ and $\eta(0)$; see Remark 7. 
Condition (24) and the internal stochastic stability of the closed-loop subsystem imply that the entry $u_{i}$ of the given controller of the form (6) solves the $H_{\infty}$ disturbance attenuation problem associated with the MJP system (25) (Li \& Ugrinovskii, 2006; Pan \& Başar, 1995). We can now apply Theorem 17 of Li \& Ugrinovskii (2006) to each system (25). This result of Li \& Ugrinovskii (2006) states that the satisfaction of (24) for the internally stable closed loop system consisting of (25) and the controller (6) is equivalent to the following conditions: The GAREs (9) admit a set of minimal positive definite definite solutions $X_{i}(j)>0, j \in \mathbb{K}$, and the GARIs (10) admit a set of positive definite solutions $Y_{i}(j)>0, j \in \mathbb{K}$ such that $Y_{i}(j)>X_{i}(j), \forall j \in \mathbb{K}$. That is, the selected collection of the constants $\tau_{i}, \theta_{i}$ belongs to the set $\mathcal{T}$, hence this set is not empty.

\subsection{Proof of the 'if' statement of Theorem 12: the sufficient condition for absolute stabi- lizability}

Suppose the set $\mathcal{T}$ is not empty. We wish to show that this property implies the absolute stabilizability of the uncertain system (1) subject to the uncertainty constraints (3) and (4). To prove this claim, we select a collection $\left\{\tau_{i}, \theta_{i}\right\}_{i=1}^{N} \in \mathcal{T}$ and show that the corresponding decentralized controller (12) defined using this collection, solves a centralized $H_{\infty}$ control problem for an aggregated MJP system comprising scaled subsystems (25) and driven by arbitrary mean-square integrable interconnection and local uncertainty inputs. In particular, this will imply that this controller is an internally stabilizing controller. Then we will show that this fact and the assumption of the theorem that the admissible uncertainty and interconnections of the system (1) satisfy the IQCs (3) and (4) together imply absolute stability of the corresponding uncertain closed loop system consisting of the plant $\mathcal{S}$ and the decentralized controller (12).

Let $\left\{\tau_{i}, \theta_{i}\right\}_{i=1}^{N} \in \mathcal{T}$ and consider the matrices $X(j)=\operatorname{diag}\left\{X_{i}(j)\right\}_{i=1}^{N}$ and $Y(j)=\operatorname{diag}\left\{Y_{i}(j)\right\}_{i=1}^{N}$ whose entries $X_{i}(j), Y_{i}(j)$ solve the corresponding GAREs (9), GARIs (10) and the coupling conditions $Y_{i}(j)>$ $X_{i}(j)$. Associated with this collection of constants, consider the system

$$
\begin{aligned}
& \dot{x}(t)=A(\eta(t)) x(t)+\bar{B}_{1}(\eta(t)) u(t)+\hat{B}_{2}(\eta(t)) \bar{w}(t), \\
& \bar{z}(t)=\tilde{C}(\eta(t)) x(t)+\tilde{D}(\eta(t)) u(t), \\
& y(t)=\tilde{\Sigma}(\eta(t)) x(t)+\tilde{\Gamma}(\eta(t)) \bar{w}(t),
\end{aligned}
$$

where

$$
\begin{aligned}
& x=\left[x_{1}^{\prime}, \cdots, x_{N}^{\prime}\right]^{\prime}, \quad u=\left[u_{1}^{\prime}, \cdots, u_{N}^{\prime}\right]^{\prime}, \quad A(j)=\operatorname{diag}\left\{A_{i}(j)\right\}_{i=1}^{N}, \\
& \bar{B}_{1}(j)=\operatorname{diag}\left\{B_{i}(j)\right\}_{i=1}^{N}, \quad \hat{B}_{2}(j)=\operatorname{diag}\left\{\bar{B}_{2, i}(j)\right\}_{i=1}^{N}, \\
& \tilde{C}(j)=\operatorname{diag}\left\{\bar{C}_{i}(j)\right\}_{i=1}^{N}, \quad \tilde{D}(j)=\operatorname{diag}\left\{\bar{D}_{i}(j)\right\}_{i=1}^{N}, \\
& \tilde{\Sigma}(j)=\operatorname{diag}\left\{\Sigma_{i}(j)\right\}_{i=1}^{N}, \quad \tilde{\Gamma}(j)=\operatorname{diag}\left\{\bar{\Gamma}_{i}(j)\right\}_{i=1}^{N}, \quad j \in \mathbb{K},
\end{aligned}
$$

in which $\bar{C}_{i}(j), \bar{D}_{i}(j), \bar{B}_{2, i}(j)$ and $\bar{\Gamma}_{i}(j)$ were defined in (11), and the input $\bar{w} \in L_{2}[0,+\infty)$. Then, we 
conclude that $X(j)>0, Y(j)>0$ satisfy the following conditions:

$$
\begin{gathered}
A^{\prime}(j) X(j)+X(j) A(j)+\sum_{v=1}^{k} q_{j v} X(v)+\tilde{C}^{\prime}(j) \tilde{C}(j) \\
-X(j)\left[\bar{B}_{1}(j) R^{-1}(j) \bar{B}_{1}^{\prime}(j)-\hat{B}_{2}(j) \hat{B}_{2}^{\prime}(j)\right] X(j)=0 \\
A^{\prime}(j) Y(j)+Y(j) A(j)+\sum_{v=1}^{k} q_{j v} Y(v)+Y(j) \hat{B}_{2}(j) \hat{B}_{2}^{\prime}(j) Y(j) \\
\quad-\left[\tilde{\Sigma}^{\prime}(j) W^{-1}(j) \tilde{\Sigma}(j)-\tilde{C}^{\prime}(j) \tilde{C}(j)\right]<0, \\
Y(j)>X(j), \\
R(j)=\operatorname{diag}\left\{R_{i}(j)\right\}_{i=1}^{N}, \quad W(j)=\operatorname{diag}\left\{W_{i}(j)\right\}_{i=1}^{N} .
\end{gathered}
$$

Consider the augmented controller $\left(A_{c}(\eta(t)), B_{c}(\eta(t)), K_{c}(\eta(t))\right)$ with state vector $x_{c}=\left[x_{c, 1}^{\prime}, \cdots, x_{c, N}^{\prime}\right]^{\prime}$, where $A_{c}(j)=\operatorname{diag}\left\{A_{c, i}(j)\right\}_{i=1}^{N}, B_{c}(j)=\operatorname{diag}\left\{B_{c, i}(j)\right\}_{i=1}^{N}, K_{c}(j)=\operatorname{diag}\left\{K_{c, i}(j)\right\}_{i=1}^{N}$ and $A_{c, i}(j), B_{c, i}(j)$, $K_{c, i}(j)$ are given by (12). Write the closed loop system as

$$
\begin{aligned}
\dot{\tilde{x}}(t) & =\mathbf{A}(\eta(t)) \tilde{x}(t)+\mathbf{B}(\eta(t)) \bar{w}(t) \\
\bar{z}(t) & =\mathbf{C}(\eta(t)) \tilde{x}(t), \\
\mathbf{A}(j) & \triangleq\left[\begin{array}{cc}
A(j) & \bar{B}_{1}(j) K_{c}(j) \\
B_{c}(j) \tilde{\Sigma}(j) & A_{c}(j)
\end{array}\right], \mathbf{B}(j) \triangleq\left[\begin{array}{c}
\hat{B}_{2}(j) \\
B_{c}(j) \tilde{\Gamma}(j)
\end{array}\right], \\
\mathbf{C}(j) & \triangleq\left[\tilde{C}(j), \tilde{D}(j) K_{c}(j)\right], \quad \tilde{x}=\left(x^{\prime}, x_{c}^{\prime}\right)^{\prime} .
\end{aligned}
$$

Assumptions 6 and 5 allow us to apply the $H_{\infty}$ control theory of MJP systems to the system (30). Using Theorem 17 of Li \& Ugrinovskii (2006) we conclude that conditions (27), (28) and (29) imply that the system (30) is internally stable and that the mapping $\mathbf{T}_{\bar{z} \bar{w}}: \bar{w} \rightarrow \bar{z}$ defined by the system (30) satisfies $\left\|\mathbf{T}_{\bar{z} \bar{w}}\right\|_{\infty}<1$. This means $\|\tilde{x}\|_{2}^{2}<\infty$ for $\tilde{x}(0)=0$ and any $\bar{w} \in L_{2}[0,+\infty)$. Then, there exists a sufficiently small $\varepsilon>0$ such that $\left\|\mathbf{T}_{\check{z} \bar{w}}\right\|_{\infty}<1$; here $\check{z} \triangleq\left[\varepsilon^{1 / 2} \tilde{x}^{\prime}, \bar{z}^{\prime}\right]^{\prime}$. By the strict bounded real lemma (e.g., see Li \& Ugrinovskii (2006, Theorem 7)), there exist $\check{\mathbf{P}}(j)>0, j=1, \cdots, k$ such that

$$
\begin{array}{r}
\check{\mathbf{P}}(j) \mathbf{A}(j)+\mathbf{A}^{\prime}(j) \check{\mathbf{P}}(j)+\sum_{v=1}^{k} q_{j v} \check{\mathbf{P}}(v) \\
+\check{\mathbf{P}}(j) \mathbf{B}(j) \mathbf{B}^{\prime}(j) \check{\mathbf{P}}(j) \\
+\mathbf{C}^{\prime}(j) \mathbf{C}(j)+\varepsilon I<0 .
\end{array}
$$

Let $\mathcal{L}$ be the extended generator associated with the jump linear system (30) (Davis, 1992). By definition,

$$
\mathcal{L}\left[\tilde{x}^{\prime} \check{\mathbf{P}}(j) \tilde{x}\right]=2 \tilde{x}^{\prime} \check{\mathbf{P}}(j)[\mathbf{A}(j) \tilde{x}+\mathbf{B}(j) \bar{w}]+\sum_{v=1}^{k} q_{j v} \tilde{x}^{\prime} \check{\mathbf{P}}(v) \tilde{x}
$$

Let $T>0$ be a time instant. Since $\check{\mathbf{P}}(j)>0$, it is easy to establish by completing the squares (Pan \& Başar, $1995)$ that for any $\bar{w} \in L_{2}[0,+\infty)$ and any initial condition $\tilde{x}(0)$, the trajectories of the closed loop system 
(30) satisfy

$$
\begin{aligned}
& \mathcal{E}\left[\int_{0}^{T}\|\check{z}(t)\|^{2} d t\right] \\
\leq & \mathcal{E}\left[\int_{0}^{T}\|\check{z}(t)\|^{2} d t\right]+\mathcal{E}\left\{\tilde{x}^{\prime}(0) \check{\mathbf{P}}(\eta(0)) \tilde{x}(0)+\int_{0}^{T} \mathcal{L}\left[\tilde{x}^{\prime}(t) \check{\mathbf{P}}(\eta(t)) \tilde{x}(t)\right] d t\right\} \\
= & \mathcal{E}\left\{\int_{0}^{T} \tilde{x}^{\prime}(t)\left(\left[\begin{array}{cc}
\tilde{C}^{\prime}(j) \tilde{C}(j) & 0 \\
0 & X(j) \bar{B}_{1}(j) R^{-1}(j) \bar{B}_{1}^{\prime}(j) X(j)
\end{array}\right]+\varepsilon I\right) \tilde{x}(t) d t\right\} \\
& +\mathcal{E}\left\{\int_{0}^{T} \tilde{x}^{\prime}(t)\left[\check{\mathbf{P}}(j) \mathbf{A}(j)+\mathbf{A}^{\prime}(j) \check{\mathbf{P}}(j)+\sum_{v=1}^{k} q_{j v} \check{\mathbf{P}}(v)\right] \tilde{x}(t) d t\right\} \\
& +\mathcal{E}\left[\int_{0}^{T} 2 \tilde{x}^{\prime}(t) \check{\mathbf{P}}(j) \mathbf{B}(j) \bar{w}(t) d t\right]+\mathcal{E}\left[\tilde{x}^{\prime}(0) \check{\mathbf{P}}(\eta(0)) \tilde{x}(0)\right] \\
\leq & \mathcal{E}\left[\tilde{x}^{\prime}(0) \check{\mathbf{P}}(\eta(0)) \tilde{x}(0)\right]-\mathcal{E}\left\{\int_{0}^{T}\left[\tilde{x}^{\prime}(t) \check{\mathbf{P}}(j) \mathbf{B}(j) \mathbf{B}^{\prime}(j) \check{\mathbf{P}}(j) \tilde{x}(t)-2 \tilde{x}^{\prime}(t) \check{\mathbf{P}}(j) \mathbf{B}(j) \bar{w}(t)\right] d t\right\} \\
= & \mathcal{E}\left[\tilde{x}^{\prime}(0) \check{\mathbf{P}}(\eta(0)) \tilde{x}(0)\right]+\mathcal{E}\left[\int_{0}^{T}\|\bar{w}(t)\|^{2} d t\right]-\mathcal{E}\left[\int_{0}^{T}\left\|\bar{w}(t)-\mathbf{B}^{\prime}(j) \check{\mathbf{P}}(j) \tilde{x}(t)\right\|^{2} d t\right] \\
\leq & \|\bar{w}\|_{2}^{2}+\mathcal{E}\left[\tilde{x}^{\prime}(0) \check{\mathbf{P}}(\eta(0)) \tilde{x}(0)\right] .
\end{aligned}
$$

Now, let $\left\{t_{l}\right\}_{l=1}^{+\infty}$ be a sequence of times as in Definitions 8 and 9. For a fixed time $t_{l}$ and an arbitrary collection of admissible $\xi_{i}, r_{i}, i=1, \cdots, N$, extend the chosen signals $\xi_{i}, r_{i}$ to have a value of zero in the interval $\left[t_{l},+\infty\right)$ to obtain $\tilde{\xi}_{i}^{l}, \tilde{r}_{i}^{l}$, and define the following uncertainty input $\bar{w}^{l}$ for the system (30):

$$
\bar{w}^{l}=\left[\tau_{1}^{1 / 2} \tilde{\xi}_{1}^{l^{\prime}}, \theta_{1}^{1 / 2} \tilde{r}_{1}^{l^{\prime}}, \cdots, \tau_{N}^{1 / 2} \tilde{\xi}_{N}^{\prime}, \theta_{N}^{1 / 2} \tilde{r}_{N}^{l^{\prime}}\right]^{\prime} .
$$

Then, $\bar{w}^{l} \in L_{2}[0,+\infty)$ and satisfies condition (32) for $T=t_{l}$. From (32), (3) and (4), we have that

$$
\begin{gathered}
\mathcal{E} \int_{0}^{t_{l}} \sum_{i=1}^{N}\left(\varepsilon\left\|\tilde{x}_{i}\right\|^{2}+\left\|z_{i}\right\|^{2}\right) d t \leq \sum_{j=1}^{k} \pi_{j} \tilde{x}^{\prime}(0) \check{\mathbf{P}}(j) \tilde{x}(0) \\
+\sum_{i=1}^{N} x_{i}^{\prime}(0)\left(\tau_{i} M_{i}+\theta_{i} \hat{M}_{i}\right) x_{i}(0)
\end{gathered}
$$

Here $z_{i}$ are the outputs of (1) corresponding to the state trajectory of the closed loop system (30) driven by the input $\bar{w}^{l}$. By definition, we can choose $t_{l} \rightarrow+\infty$. Then (34) implies $x_{i}, x_{c, i}, u_{i} \in L_{2}[0,+\infty)$. Consequently, $\xi_{i}, r_{i}, \zeta_{i} \in L_{2}[0,+\infty)$. Then, condition (7) follows from (34), (3) and (4). This proves absolute stability of the closed loop system.

Remark 14. Although in Theorem 12 we focus on the class of controllers which have the decentralized structure (6), the result of this theorem can be extended to a more general class of LTI centralized controllers. In fact, it is possible to show using an approach similar to the one used in the above proofs that the system $\mathcal{S}$ under consideration can be stabilized by a decentralized controller of the form (6) if and only if it can be stabilized by a centralized LTI controller. The proof is based on the observation made above that the decentralized absolutely stabilizing controller under consideration, which solves the decoupled $H_{\infty}$ control problem, also solves the corresponding $H_{\infty}$ control problem for the aggregated system. The proof of the 'only if' part of Theorem 12 can also be modified to show that if the $H_{\infty}$ control problem for the aggregated system admits a solution, then it can be solved via decentralized control. We refer to Petersen et al. (2000) for a detailed discussion of this issue. 


\subsection{Proof of Theorem 13}

Select $\left\{\tau_{i}, \theta_{i}\right\}_{i=1}^{N} \in \mathcal{T}$ and, using the solutions to the corresponding GAREs (9) and GARIs (10), define $\Phi(j)=\left[\begin{array}{cc}Y(j) & X(j)-Y(j) \\ X(j)-Y(j) & Y(j)-X(j)\end{array}\right]$. It is easy to show that the matrices $\Phi(j), j \in \mathbb{K}$ satisfy the following GARIs (Pan \& Başar, 1995) for every $j \in \mathbb{K}$ :

$$
\begin{aligned}
& \Phi(j) \mathbf{A}(j)+\mathbf{A}^{\prime}(j) \Phi(j)+\Phi(j) \mathbf{B}(j) \mathbf{B}^{\prime}(j) \Phi(j)+\mathbf{C}^{\prime}(j) \mathbf{C}(j) \\
& +\sum_{v=1}^{k} q_{j v} \Phi(v)=\left[\begin{array}{cc}
R_{Y}(j) & R_{X}(j)-R_{Y}(j) \\
R_{X}(j)-R_{Y}(j) & R_{Y}(j)-R_{X}(j)
\end{array}\right] \leq 0
\end{aligned}
$$

here $\mathbf{A}(j), \mathbf{B}(j)$ and $\mathbf{C}(j)$ are coefficients of the modes of the closed loop system (30) corresponding to the controller (6), (12), and $R_{X}(j), R_{Y}(j)$ denote the left-hand side of (27) and (28) respectively. Note that $R_{X}(j)=0$.

Now, choose $\tilde{x}^{\prime}(t) \Phi(\eta(t)) \tilde{x}(t)$ as a candidate Lyapunov function. Then, similar to (31), (32) and (34), we have

$$
\mathcal{E}\left[\int_{0}^{T}\|\bar{z}(t)\|^{2} d t\right] \leq\|\bar{w}\|_{2}^{2}+\mathcal{E}\left[\tilde{x}^{\prime}(0) \Phi(\eta(0)) \tilde{x}(0)\right]
$$

and

$$
\mathcal{E} \int_{0}^{+\infty} \sum_{i=1}^{N}\left\|z_{i}\right\|^{2} d t \leq \sum_{j=1}^{k} \pi_{j} \tilde{x}^{\prime}(0) \Phi(j) \tilde{x}(0)+\sum_{i=1}^{N} x_{i}^{\prime}(0)\left(\tau_{i} M_{i}+\theta_{i} \hat{M}_{i}\right) x_{i}(0),
$$

where $\bar{z}(t)$ is the output of the closed loop system (30), and $z_{i}(\cdot)$ are the outputs of (1) corresponding to the state trajectory of the closed loop system (30) driven by the input $\bar{w}^{l}(\cdot)$ in (33). Therefore,

$$
\begin{gathered}
J_{w c}(u) \leq \sum_{j=1}^{k} \pi_{j} \tilde{x}^{\prime}(0) \Phi(j) \tilde{x}(0)+\sum_{i=1}^{N} x_{i}^{\prime}(0)\left(\tau_{i} M_{i}+\theta_{i} \hat{M}_{i}\right) x_{i}(0) \\
=\sum_{j=1}^{k} \pi_{j}\left[x^{\prime}(0) X(j) x(0)+\left(x_{c}(0)-x(0)\right)^{\prime}(Y(j)-X(j))\right. \\
\left.\left(x_{c}(0)-x(0)\right)\right]+\sum_{i=1}^{N} x_{i}^{\prime}(0)\left(\tau_{i} M_{i}+\theta_{i} \hat{M}_{i}\right) x_{i}(0) .
\end{gathered}
$$

It is obvious from (36) that letting $x_{c}(0)=x(0)$ provides the smallest bound on the worst-case performance:

$$
J_{w c}(u) \leq J(\tau, \theta)
$$

Now substituting $\tau_{i}=\tau_{i}^{*}, \theta_{i}=\theta_{i}^{*}, i=1, \cdots, N$ into (37) yields (14). Then (15) follows from (14) trivially.

\section{Computational Algorithm}

\subsection{A rank constrained LMI realization}

As shown in Theorem 13, the proposed suboptimal decentralized output feedback controller design involves solving the optimization problem given on the right-hand side of $(15), \inf _{\mathcal{T}} J(\tau, \theta)$. Generally, it is difficult to provide a systematic way to perform such optimization. In this section, we discuss one possible approach to address this difficulty numerically. The idea is to replace the problem $\inf _{\mathcal{T}} J(\tau, \theta)$ with an equivalent optimization problem involving rank constrained LMIs. First we introduce a related rank constrained LMI optimization problem. Next we prove the equivalence between the two problems; see Theorem 15 given below. 
To introduce the rank constrained LMI optimization problem related to the optimization problem stated in Theorem 13, similar to Ait Rami \& El Ghaoui (1996), consider the following inequalities instead of the Riccati equations (9),

$$
\begin{aligned}
& A_{i}^{\prime}(j) X_{i}(j)+X_{i}(j) A_{i}(j)+\sum_{v=1}^{k} q_{j v} X_{i}(v)+\bar{C}_{i}^{\prime}(j) \bar{C}_{i}(j) \\
& -X_{i}(j)\left[B_{i}(j) R_{i}^{-1}(j) B_{i}^{\prime}(j)-\bar{B}_{2, i}(j) \bar{B}_{2, i}^{\prime}(j)\right] X_{i}(j)<0 .
\end{aligned}
$$

By left and right multiplying (38) with $\tilde{X}_{i}(j)=X_{i}^{-1}(j)$, we obtain

$$
\begin{aligned}
\tilde{X}_{i}(j) & A_{i}^{\prime}(j)+A_{i}(j) \tilde{X}_{i}(j)+\sum_{v=1}^{k} q_{j v} \tilde{X}_{i}(j) \tilde{X}_{i}^{-1}(v) \tilde{X}_{i}(j) \\
& +\tilde{X}_{i}(j) \bar{C}_{i}^{\prime}(j) \bar{C}_{i}(j) \tilde{X}_{i}(j)-\left[B_{i}(j) R_{i}^{-1}(j) B_{i}^{\prime}(j)-\bar{B}_{2, i}(j) \bar{B}_{2, i}^{\prime}(j)\right]<0 .
\end{aligned}
$$

Introducing matrices $F_{i}(j)$ of appropriate dimensions, without changing the feasibility of (39), we add a quadratic term of $F_{i}(j)$ to the left-hand side of (39) as follows,

$$
\begin{aligned}
\tilde{X}_{i}(j) A_{i}^{\prime}(j) & +A_{i}(j) \tilde{X}_{i}(j)+\sum_{v=1}^{k} q_{j v} \tilde{X}_{i}(j) \tilde{X}_{i}^{-1}(v) \tilde{X}_{i}(j) \\
& +\tilde{X}_{i}(j) \bar{C}_{i}^{\prime}(j) \bar{C}_{i}(j) \tilde{X}_{i}(j)-\left[B_{i}(j) R_{i}^{-1}(j) B_{i}^{\prime}(j)-\bar{B}_{2, i}(j) \bar{B}_{2, i}^{\prime}(j)\right] \\
& +\left[F_{i}^{\prime}(j)+B_{i}(j) R_{i}^{-1}(j)\right] R_{i}(j)\left[F_{i}^{\prime}(j)+B_{i}(j) R_{i}^{-1}(j)\right]^{\prime}<0
\end{aligned}
$$

which is

$$
\begin{aligned}
\tilde{X}_{i}(j) & A_{i}^{\prime}(j)+A_{i}(j) \tilde{X}_{i}(j)+\sum_{v=1}^{k} q_{j v} \tilde{X}_{i}(j) \tilde{X}_{i}^{-1}(v) \tilde{X}_{i}(j)+\tilde{X}_{i}(j) \bar{C}_{i}^{\prime}(j) \bar{C}_{i}(j) \tilde{X}_{i}(j) \\
& +\bar{B}_{2, i}(j) \bar{B}_{2, i}^{\prime}(j)+F_{i}^{\prime}(j) R_{i}(j) F_{i}(j)+B_{i}(j) F_{i}(j)+F_{i}^{\prime}(j) B_{i}^{\prime}(j)<0 .
\end{aligned}
$$

Using (11), the terms of (40) can be represented as follows,

$$
\begin{aligned}
\bar{B}_{2, i}(j) \bar{B}_{2, i}^{\prime}(j) & =\tau_{i}^{-1} E_{i}(j) E_{i}^{\prime}(j)+\theta_{i}^{-1} L_{i}(j) L_{i}^{\prime}(j), \\
\tilde{X}_{i}(j) \bar{C}_{i}^{\prime}(j) \bar{C}_{i}(j) \tilde{X}_{i}(j) & =\tilde{X}_{i}(j)\left[C_{i}^{\prime}(j) C_{i}(j)+\left(\tau_{i}+\bar{\theta}_{i}\right) H_{i}^{\prime}(j) H_{i}(j)\right] \tilde{X}_{i}(j), \\
F_{i}^{\prime}(j) R_{i}(j) F_{i}(j) & =F_{i}^{\prime}(j)\left[D_{i}^{\prime}(j) D_{i}(j)+\left(\tau_{i}+\bar{\theta}_{i}\right) G_{i}^{\prime}(j) G_{i}(j)\right] F_{i}(j) .
\end{aligned}
$$

Let $\tilde{\tau}_{i}=\tau_{i}^{-1}, \tilde{\theta}_{i}=\theta_{i}^{-1}$. By combining (40), (41) and applying the Schur complement, we obtain the following LMIs in the variables $\tilde{X}_{i}(j), F_{i}(j), \tilde{\tau}_{i}, \tilde{\theta}_{i}$ :

$$
\left[\begin{array}{cccc}
\mathbf{N}_{i}(j) & \mathbf{V}_{i}(j) & F_{i}^{\prime}(j) D_{i}^{\prime}(j)+\tilde{X}_{i}(j) C_{i}^{\prime}(j) & \mathbf{Q}_{i}(j) \\
\star & -\mathbf{S}_{i}(j) & \mathbf{0} & \mathbf{0} \\
\star & \star & -\mathbf{I} & \mathbf{0} \\
\star & \star & \star & -\boldsymbol{\Theta}_{i}
\end{array}\right]<0
$$

where

$$
\begin{aligned}
& \mathbf{N}_{i}(j)=\tilde{X}_{i}(j) A_{i}^{\prime}(j)+A_{i}(j) \tilde{X}_{i}(j)+q_{j j} \tilde{X}_{i}(j)+B_{i}(j) F_{i}(j) \\
& +F_{i}^{\prime}(j) B_{i}^{\prime}(j)+\tilde{\tau}_{i} E_{i}(j) E_{i}^{\prime}(j)+\tilde{\theta}_{i} L_{i}(j) L_{i}^{\prime}(j), \\
& \mathbf{V}_{i}(j)=\left[\sqrt{q_{j 1}} \tilde{X}_{i}(j) \cdots \sqrt{q_{j(j-1)}} \tilde{X}_{i}(j),\right. \\
& \left.\sqrt{q_{j(j+1)}} \tilde{X}_{i}(j) \cdots \sqrt{q_{j k}} \tilde{X}_{i}(j)\right], \\
& \mathbf{S}_{i}(j)=\operatorname{diag}\left[\tilde{X}_{i}(1) \cdots \tilde{X}_{i}(j-1), \tilde{X}_{i}(j+1) \cdots \tilde{X}_{i}(k)\right], \\
& \mathbf{Q}_{i}(j)=\left[F_{i}^{\prime}(j) G_{i}^{\prime}(j)+\tilde{X}_{i}(j) H_{i}^{\prime}(j), \cdots,\right. \\
& \left.F_{i}^{\prime}(j) G_{i}^{\prime}(j)+\tilde{X}_{i}(j) H_{i}^{\prime}(j)\right] \text { ( } N \text { entries), } \\
& \boldsymbol{\Theta}_{i}=\operatorname{diag}\left[\tilde{\tau}_{i} \mathbf{I}, \tilde{\theta}_{1} \mathbf{I}, \cdots, \tilde{\theta}_{i-1} \mathbf{I}, \tilde{\theta}_{i+1} \mathbf{I}, \cdots, \tilde{\theta}_{N} \mathbf{I}\right] .
\end{aligned}
$$


In a similar fashion, the GARIs (10) can be transformed into the following equivalent LMIs in the variables $Y_{i}(j), \tau_{i}, \theta_{i}$ :

$$
\left[\begin{array}{ccc}
\mathbf{M}_{i}(j) & Y_{i}(j) E_{i}(j) & Y_{i}(j) L_{i}(j) \\
\star & -\tau_{i} \mathbf{I} & \mathbf{0} \\
\star & \star & -\theta_{i} \mathbf{I}
\end{array}\right]<0,
$$

where

$$
\begin{gathered}
\mathbf{M}_{i}(j)=A_{i}^{\prime}(j) Y_{i}(j)+Y_{i}(j) A_{i}(j)+\sum_{v=1}^{k} q_{j v} Y_{i}(v)+C_{i}^{\prime}(j) C_{i}(j) \\
-\tau_{i} \Sigma_{i}^{\prime}(j)\left[\Gamma_{i}(j) \Gamma_{i}^{\prime}(j)\right]^{-1} \Sigma_{i}(j)+\left(\tau_{i}+\bar{\theta}_{i}\right) H_{i}^{\prime}(j) H_{i}(j) .
\end{gathered}
$$

The coupling condition $Y_{i}(j)>X_{i}(j)>0$ is equivalent to

$$
\left[\begin{array}{cc}
\tilde{X}_{i}(j) & \mathbf{I} \\
\mathbf{I} & Y_{i}(j)
\end{array}\right]>0
$$

Next, consider the performance upper bound given by the right-hand side of (15). Note that minimizing $J(\tau, \theta)$ is equivalent to minimizing $\lambda_{1}+\cdots+\lambda_{N}$ subject to

$$
\lambda_{i}>x_{i 0}^{\prime} \Psi_{i}\left(X_{i}, \tau_{i}, \theta_{i}\right) x_{i 0}, \quad i=1, \cdots, N .
$$

By Schur complement, (45) is equivalent to the LMI

$$
\left[\begin{array}{cccc}
\lambda_{i} & \boldsymbol{\Pi}_{i} & x_{i 0}^{\prime} M_{i}^{1 / 2} & x_{i 0}^{\prime} \hat{M}_{i}^{1 / 2} \\
\star & \tilde{\mathbf{X}}_{i} & \mathbf{0} & \mathbf{0} \\
\star & \star & \tilde{\tau}_{i} \mathbf{I} & \mathbf{0} \\
\star & \star & \star & \tilde{\theta}_{i} \mathbf{I}
\end{array}\right]>0, \quad i=1, \cdots, N ;
$$

here $\Pi_{i}=\left[\pi_{1}^{1 / 2} x_{i 0}^{\prime}, \cdots, \pi_{k}^{1 / 2} x_{i 0}^{\prime}\right], \quad \tilde{\mathbf{X}}_{i}=\operatorname{diag}\left\{\tilde{X}_{i}(j)\right\}_{j=1}^{k}$.

Also, the conditions $\tilde{\tau}_{i}>0, \tau_{i}>0, \tilde{\tau}_{i} \tau_{i}=1, \tilde{\theta}_{i}>0, \theta_{i}>0, \tilde{\theta}_{i} \theta_{i}=1$ are equivalent to the rank constrained LMIs

$$
\begin{aligned}
& {\left[\begin{array}{cc}
\tilde{\tau}_{i} & 1 \\
1 & \tau_{i}
\end{array}\right] \geq 0, \quad \operatorname{rank}\left[\begin{array}{cc}
\tilde{\tau}_{i} & 1 \\
1 & \tau_{i}
\end{array}\right] \leq 1,} \\
& {\left[\begin{array}{cc}
\tilde{\theta}_{i} & 1 \\
1 & \theta_{i}
\end{array}\right] \geq 0, \quad \operatorname{rank}\left[\begin{array}{cc}
\tilde{\theta}_{i} & 1 \\
1 & \theta_{i}
\end{array}\right] \leq 1 .}
\end{aligned}
$$

We now consider the following optimization problem in the variables $\lambda_{i}, \tilde{X}_{i}(j), F_{i}(j), Y_{i}(j), \tilde{\tau}_{i}, \tilde{\theta}_{i}$, and $\tau_{i}, \theta_{i}:$ Find

$$
\begin{aligned}
& J_{L M I}^{*} \triangleq \inf \left(\lambda_{1}+\cdots+\lambda_{N}\right) \\
& \text { subject to (42), (43), (44), (46) and (47). }
\end{aligned}
$$

Note that this problem consists of minimizing a linear cost subject to rank constrained LMIs. To solve this problem in our numerical experiments, we use the rank constrained LMI solver LMIRank. We postpone discussion of this algorithm until Section 5.2. Meanwhile, we show that the optimization problem (48) and the optimization problem on the right hand side of (15) are equivalent.

Theorem 15. Let $J_{R i c}^{*} \triangleq \inf _{\mathcal{T}} J(\tau, \theta)$, and $J_{L M I}^{*}$ be defined as in (48). Then $J_{R i c}^{*}=J_{L M I}^{*}$. 
Proof. Suppose (48) has a feasible solution $\lambda_{i}, \tilde{X}_{i}^{+}(j), F_{i}(j), Y_{i}(j), \tilde{\tau}_{i}, \tilde{\theta}_{i}, \tau_{i}$ and $\theta_{i}$. Then $X_{i}^{+}(j)=$ $\left[\tilde{X}_{i}^{+}(j)\right]^{-1}$ satisfies (38) and $Y_{i}(j)>X_{i}^{+}(j)$. By Corollary 3.1 of Pan \& Başar (1995), there exist $X_{i}(j)>0$ satisfying (9) such that $X_{i}(j)<X_{i}^{+}(j)$. As $X_{i}(j), Y_{i}(j)$ satisfy the GAREs (9) and GARIs (10), and $Y_{i}(j)>X_{i}^{+}(j)>X_{i}(j)$, we have $\left\{\tau_{i}, \theta_{i}\right\}_{i=1}^{N} \in \mathcal{T}$. This implies that the set of $\left\{\tau_{i}, \theta_{i}\right\}_{i=1}^{N}$ for which the constraints of the problem (48) can be satisfied is a subset of the set $\mathcal{T}$. Also, for those $\left\{\tau_{i}, \theta_{i}\right\}_{i=1}^{N}$, the fact $X_{i}^{+}(j)>X_{i}(j)$ implies

$$
J(\tau, \theta) \leq \sum_{i=1}^{N} x_{i 0}^{\prime} \Psi_{i}\left(X_{i}^{+}, \tau_{i}, \theta_{i}\right) x_{i 0}<\sum_{i=1}^{N} \lambda_{i}
$$

see (13) and (45). Hence, $J_{R i c}^{*} \leq J_{L M I}^{*}$.

Conversely, for any $\varepsilon>0$, there exist $\left\{\tau_{i}, \theta_{i}\right\}_{i=1}^{N} \in \mathcal{T}, X_{i}(j)$ and $Y_{i}(j)$ verifying GAREs (9), GARIs (10) with $Y_{i}(j)>X_{i}(j)$, such that

$$
J_{R i c}^{*}+\varepsilon>J(\tau, \theta)=\sum_{i=1}^{N} x_{i 0}^{\prime} \Psi_{i}\left(X_{i}, \tau_{i}, \theta_{i}\right) x_{i 0} .
$$

By Corollary 3.1 of Pan \& Başar (1995), for an arbitrarily small $\delta>0$, there exist $X_{i}^{+}(j)>0$ satisfying (38) such that $X_{i}^{+}(j)>X_{i}(j)>X_{i}^{+}(j)-\delta I, j \in \mathbb{K}, i=1, \cdots, N$. Furthermore, we can select $\delta$ sufficiently small so that $Y_{i}(j)>X_{i}(j)+\delta I>X_{i}^{+}(j)$. Therefore, since $\sum_{j=1}^{k} \pi_{j}=1$, then

$$
J_{R i c}^{*}+\varepsilon>\sum_{i=1}^{N} x_{i 0}^{\prime} \Psi_{i}\left(X_{i}^{+}, \tau_{i}, \theta_{i}\right) x_{i 0}-\delta \sum_{i=1}^{N}\left\|x_{i 0}\right\|^{2} .
$$

Let $\tilde{X}_{i}^{+}(j)=\left[X_{i}^{+}(j)\right]^{-1}, F_{i}(j)=-R_{i}^{-1}(j) B_{i}^{\prime}(j), \tilde{\tau}_{i}=\tau_{i}^{-1}, \tilde{\theta}_{i}=\theta_{i}^{-1}$, and $\lambda_{i}=\rho+x_{i 0}^{\prime} \Psi_{i}\left(X_{i}^{+}, \tau_{i}, \theta_{i}\right) x_{i 0}$ where $\rho>0$ is any positive constant. Then $\tilde{X}_{i}^{+}(j), F_{i}(j), Y_{i}(j), \tilde{\tau}_{i}, \tilde{\theta}_{i}, \tau_{i}, \theta_{i}$ and $\lambda_{i}$ satisfy conditions (42), (43), (44), (46) and (47). Therefore,

$$
J_{L M I}^{*} \leq \sum_{i=1}^{N} \lambda_{i}<N \rho+J_{R i c}^{*}+\varepsilon+\delta \sum_{i=1}^{N}\left\|x_{i 0}\right\|^{2} .
$$

Letting $\rho, \varepsilon, \delta \rightarrow 0$, we have $J_{L M I}^{*} \leq J_{R i c}^{*}$. This completes the proof.

The state feedback control design result of Ugrinovskii \& Pota (2005) only uses GAREs (9). GARIs (10) and the LMIs (43), (44) and conditions (47) do not arise in this case. Therefore, the state feedback problem reduces to the following convex semidefinite programming problem in the variables $\tilde{X}_{i}(j), F_{i}(j)$, $\tilde{\tau}_{i}, \tilde{\theta}_{i}, \lambda_{i}$ :

$$
\inf \left(\lambda_{1}+\cdots+\lambda_{N}\right) \text { subject to (42) and (46). }
$$

A globally optimal controller, if it exists, can be obtained by solving (50). On the other hand, if the solution to (50) does not exist, then the system has infinitely large minimax optimal performance. This is an indication that the system can be driven unstable by one of the admissible uncertainties or interconnections. Hence, the system is not absolutely stabilizable; see Ugrinovskii \& Pota (2005) for details.

A control design methodology based on Theorem 15 is as follows.

- Solve the rank constrained LMI problem (48), to a desired accuracy, obtaining a collection $\theta_{i}, \tau_{i}$, $\tilde{X}_{i}^{+}(j), Y_{i}(j)$. It follows from the proof of Theorem 15 that $\left\{\theta_{i}, \tau_{i}\right\}_{i=1}^{N}$ belongs to the feasibility set $\mathcal{T}$.

- Substitute the found $\theta_{i}, \tau_{i}$ into the GAREs (9) and solve (9) to obtain $X_{i}(j)$. Note that $Y_{i}(j)$ can be selected from the found feasible solution to the problem (48); see the proof of Theorem 15.

- Construct the robust stabilizing controller (6), (12) using the found $\theta_{i}, \tau_{i}, X_{i}(j)$ and $Y_{i}(j)$. Also, $J(\tau, \theta)$ can be computed. 
Going from the auxiliary rank constrained optimization problem (48) back to the underlying robust control problem, we summarize the relation between the costs of the three optimization problems involved in the control design algorithm,

$$
J_{w c}(u) \leq J(\tau, \theta)<\sum_{i=1}^{N} \lambda_{i}
$$

The first quantity is the true worst-case performance cost associated with the obtained robust stabilizing controller; this is the cost of the optimization problem on the left hand side of (15). The second quantity is the cost of the optimization problem defined on the right hand side of (15). As shown (see (37)), it provides an upper bound on the worst-case performance associated with this controller and, unlike $J_{w c}(u)$, can be computed explicitly. The third quantity is the cost of the auxiliary rank constrained optimization problem (48). The relation between this quantity and $J(\tau, \theta)$ follows from (49). Hence the solution of the optimization problem (48) also provides an upper bound on robust performance of the control system which, as we see, is more conservative than that given by $J(\tau, \theta)$.

\subsection{LMIRank toolbox for rank constrained LMIs}

LMIRank (Orsi, 2005) is a MATLAB toolbox for solving feasibility problems defined in terms of LMIs such as

$$
F(x) \triangleq F_{0}+\sum_{i=1}^{m} x_{i} F_{i} \geq 0
$$

and rank constrained LMIs of the form

$$
G(x) \triangleq G_{0}+\sum_{i=1}^{m} x_{i} G_{i} \geq 0, \quad \operatorname{rank} G(x) \leq r .
$$

Multiple constraints of either type can be specified. It is an implementation of a Newton-like method presented in Orsi et al. (2006).

The constraints in problem (48) are exactly of the types mentioned above. As problem (48) is an optimization problem (with a linear cost) rather than a feasibility problem, LMIRank can be used to solve the problem via a standard bisection procedure.

As mentioned before, currently available numerical methods for solving rank constrained LMI problems are not guaranteed to always find a solution. Hence, for an optimization problem involving rank constrained LMIs it may not always be possible to find a solution whose cost is near the minimum cost achievable. Despite this, some of the currently available methods, such as LMIRank, perform well in practice. A demonstration of this fact is given in Section 7.

\section{Stable modes}

We now show that, by using the same method as that in Mariton \& Bertrand (1985) and Ugrinovskii \& Pota (2005), the mode of each subsystem can also be made robustly asymptotically stable while the robust stochastic stability of the closed loop system is preserved. Recall that $q_{j j} \leq 0$. In this section, we will assume for simplicity ${ }^{1}$ that $q_{j j}<0, \forall j \in \mathbb{K}$.

\footnotetext{
${ }^{1}$ This assumption can be easily removed by noting that if $q_{j j}=0$, then $q_{j v}=0 \forall v \in \mathbb{K}$, and hence the state $j$ of the underlying Markov chain is absorbing; i.e., the system will remain in this mode with probability 1. Furthermore, the GAREs (9) and GARIs (10) become standard AREs and ARIs arising in the deterministic robust control theory. Hence the stability of the absorbing mode can be analyzed using the existing theory.
} 
Consider the following collection of GAREs and GARIs:

$$
\begin{aligned}
& A_{i}^{\prime}(j) X_{i}(j)+X_{i}(j) A_{i}(j)+\sum_{v \neq j} q_{j v} X_{i}(v)+\bar{C}_{i}^{\prime}(j) \bar{C}_{i}(j) \\
& -X_{i}(j)\left[B_{i}(j) R_{i}^{-1}(j) B_{i}^{\prime}(j)-\bar{B}_{2, i}(j) \bar{B}_{2, i}^{\prime}(j)\right] X_{i}(j)=0, \\
& A_{i}^{\prime}(j) Y_{i}(j)+Y_{i}(j) A_{i}(j)+Y_{i}(j) \bar{B}_{2, i}(j) \bar{B}_{2, i}^{\prime}(j) Y_{i}(j) \\
& -\left[\Sigma_{i}^{\prime}(j) W_{i}^{-1}(j) \Sigma_{i}(j)-\bar{C}_{i}^{\prime}(j) \bar{C}_{i}(j)\right]+\sum_{v \neq j} q_{j v} Y_{i}(v)<0 .
\end{aligned}
$$

Let $\check{\mathcal{T}}$ denote the collection of constants $\check{\tau}_{i}, \check{\theta}_{i}$ such that (51) and (52) admit positive definite solutions $\check{X}_{i}(j)$, $\check{Y}_{i}(j), j=1, \cdots, k$ satisfying $\check{Y}_{i}(j)>\check{X}_{i}(j), \forall j \in \mathbb{K}$. If the set $\check{\mathcal{T}}$ is non-empty, let $\check{\mathcal{u}}$ be the class of decentralized controllers (6), (12) in which the matrices $X_{i}(j), Y_{i}(j)$ are replaced by $\check{X}_{i}(j), \breve{Y}_{i}(j)$, respectively. Then we have the following result:

Theorem 16. Under conditions of Theorem 12, if the set $\check{\mathcal{T}}$ is not empty, the system $\mathcal{S}$ is absolutely stabilizable using any decentralized dynamic output feedback controller in $\breve{u}$. In addition, any controller in $\breve{u}$ renders deterministic modes of the closed-loop system robustly stable.

Furthermore, given a vector of initial conditions $x_{i}(0)=x_{i 0}$, let $\left\{\check{\tau}_{i}^{*}, \check{\theta}_{i}^{*}\right\}_{i=1}^{N} \in \check{\mathcal{T}}$ attain the infimum

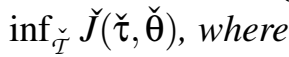

$$
\check{J}(\check{\tau}, \check{\theta}) \triangleq \sum_{i=1}^{N} x_{i 0}^{\prime} \Psi_{i}\left(\check{X}_{i}, \check{\tau}_{i}, \check{\theta}_{i}\right) x_{i 0}
$$

Then a decentralized controller $\breve{u}^{*}$ whose worst-case performance satisfies the upper bound

$$
J_{w c}\left(\check{u}^{*}\right) \leq \inf _{\breve{\tau}} \check{J}(\check{\tau}, \check{\theta}),
$$

is given by (6), (12) in which $\check{\tau}_{i}=\check{\tau}_{i}^{*}, \check{\theta}_{i}=\check{\theta}_{i}^{*}, i=1, \cdots, N, X_{i}(j), Y_{i}(j)$ are replaced by $\check{X}_{i}(j), \check{Y}_{i}(j)$, respectively, with the initial condition $x_{c, i}(0)=x_{i}(0)$.

Proof. Let $\left\{\check{\tau}_{i}, \check{\theta}_{i}\right\}_{i=1}^{N} \in \check{\mathcal{T}}$ and consider a collection of positive definite symmetric matrices $\left\{\check{X}_{i}(j)\right\}_{i, j=1}^{N}$, $\left\{\check{Y}_{i}(j)\right\}_{i, j=1}^{N}$ which satisfy (51), (52), such that $\check{Y}_{i}(j)>\check{X}_{i}(j), \forall j \in \mathbb{K}$. Then, it is easy to see that

$$
\begin{aligned}
A_{i}^{\prime}(j) \check{X}_{i}(j) & +\check{X}_{i}(j) A_{i}(j)+\bar{C}_{i}^{\prime}(j) \bar{C}_{i}(j) \\
& -\check{X}_{i}(j)\left[B_{i}(j) R_{i}^{-1}(j) B_{i}^{\prime}(j)-\bar{B}_{2, i}(j) \bar{B}_{2, i}^{\prime}(j)\right] \check{X}_{i}(j)+\sum_{v=1}^{k} q_{j v} \check{X}_{i}(v)<0, \\
A_{i}^{\prime}(j) \check{Y}_{i}(j)+\check{Y}_{i}(j) A_{i}(j) & +\check{Y}_{i}(j) \bar{B}_{2, i}(j) \bar{B}_{2, i}^{\prime}(j) \check{Y}_{i}(j) \\
& -\left[\Sigma_{i}^{\prime}(j) W_{i}^{-1}(j) \Sigma_{i}(j)-\bar{C}_{i}^{\prime}(j) \bar{C}_{i}(j)\right]+\sum_{v=1}^{k} q_{j v} \check{Y}_{i}(v)<0 .
\end{aligned}
$$

It can then be readily shown using the argument of the proof of Theorem 12 that the decentralized controller $\check{u}$ of the form (12) in which matrices $X_{i}(j), Y_{i}(j)$ are replaced by $\breve{X}_{i}(j), \breve{Y}_{i}(j)$, respectively, solves the robust decentralized stabilization problem. To established this claim we only need to verify that the GARIs (35) still hold for the solutions $\check{X}_{i}(j), \breve{Y}_{i}(j)$ to the GARIs (51), (52) with $\breve{Y}_{i}(j)>\check{X}_{i}(j), \forall j \in \mathbb{K}$. Similarly to (27), (28), let $R_{\check{X}}(j), R_{\check{X}}(j)$ denote the left-hand side of the stacked equations (54), (55) respectively, and let $\check{X}(j), \check{Y}(j)$ be defined similarly. By (51) and (52), we have $R_{\check{X}}(j)=q_{j j} \check{X}(j)<0$ and $R_{\breve{Y}}(j)-q_{j j} \check{Y}(j)<0$. Then

$$
R_{\check{Y}}(j)-R_{\check{X}}(j)=R_{\breve{Y}}(j)-q_{j j} \check{Y}(j)+q_{j j}[\check{Y}(j)-\check{X}(j)]<0,
$$


therefore the GARIs (35) follow using the Schur complement, as required. This further implies that the corresponding closed-loop system is internally stochastically stable and satisfies the $H_{\infty}$ norm condition (23); see the proof of the implication $(i i) \Rightarrow(i)$ in Li \& Ugrinovskii (2006, Theorem 17).

Also, it follows from the GAREs (51) and GARIs (52) that

$$
\begin{aligned}
& A_{i}^{\prime}(j) \check{X}_{i}(j)+\check{X}_{i}(j) A_{i}(j)+\bar{C}_{i}^{\prime}(j) \bar{C}_{i}(j) \\
& -\check{X}_{i}(j)\left[B_{i}(j) R_{i}^{-1}(j) B_{i}^{\prime}(j)-\bar{B}_{2, i}(j) \bar{B}_{2, i}^{\prime}(j)\right] \check{X}_{i}(j)<0, \\
& A_{i}^{\prime}(j) \check{Y}_{i}(j)+\check{Y}_{i}(j) A_{i}(j)+\check{Y}_{i}(j) \bar{B}_{2, i}(j) \bar{B}_{2, i}^{\prime}(j) \check{Y}_{i}(j) \\
& -\left[\Sigma_{i}^{\prime}(j) W_{i}^{-1}(j) \Sigma_{i}(j)-\bar{C}_{i}^{\prime}(j) \bar{C}_{i}(j)\right]<0 .
\end{aligned}
$$

Together with the condition $\check{Y}_{i}(j)>\check{X}_{i}(j), \forall j \in \mathbb{K}$, these inequalities imply that the controller considered in this section is a robust stabilizing controller for each mode of the uncertain system $\mathcal{S}$ corresponding to one of the states of the Markov chain $\eta(t)$ (Dullerud \& Paganini, 2000).

Furthermore, similarly to (36), the considered controller with $x_{c, i}(0)=x_{i}(0)$ yields the following bound on the worst case performance cost:

$$
J_{w c}(\check{u}) \leq \check{J}(\check{\tau}, \check{\theta})
$$

Substituting $\check{\tau}_{i}=\check{\tau}_{i}^{*}, \check{\theta}_{i}=\check{\theta}_{i}^{*}, i=1, \cdots, N$ into (56) yields (53). This completes the proof.

Note that to solve (51) and (52) numerically, a rank constrained LMI problem similar to that considered in the previous section can be introduced. This can be done by removing the terms corresponding to $q_{j j}$ from $\mathbf{N}_{i}(j)$ and $\mathbf{M}_{i}(j)$; see equations (42) and (43).

\section{Example}

We consider a 9-bus power system consisting of four subsystems: three generators and one on-load tap changing transformer (OLTC). The system diagram and numerical parameters for the system are presented in the Appendix. A complete mathematical description of the system and the derivation of the state equations are given in Pota, Athanasius, Ugrinovskii, \& Li (2006). It is shown in that reference that the linearized state equation consists of four subsystems of the form of (1). The three 2nd order subsystems $S_{1}, S_{2}, S_{3}$ describe generator dynamics and the 1 st order subsystem $S_{4}$ describes those of the OLTC device. The state variables $x_{1}, x_{2}$ and $x_{3}$ describe the change in the generator rotor angles relative the reference frame and the corresponding changes in the generated frequency relative the reference $50 \mathrm{~Hz}$. The variable $x_{4}$ describes the change in the OLTC transformation ratio.

In Pota et al. (2006), linear models are obtained for two different steady-state load conditions on the power system. System transitions between the above two load conditions are described by the Markov chain parameter $\eta(t)$ whose transition probabilities are determined by the parameters $q_{12}=q_{21}=0.1$, and the initial distribution is $\pi=\left[\begin{array}{ll}0.5 & 0.5\end{array}\right]^{\prime}$. Parameters of the system model (1) corresponding to the two load profiles under consideration are given in the Appendix. To design the controllers we let

$$
\begin{aligned}
& C_{i}(j)=\left[\begin{array}{c}
0.1 \mathbf{I}_{2} \\
\mathbf{0}_{2}
\end{array}\right], i=1,2,3, \quad C_{4}(j)=\left[\begin{array}{c}
0.1 \\
0
\end{array}\right], j=1,2, \\
& D_{i}(j)=\left[\begin{array}{c}
\mathbf{0}_{2} \\
0.1 \mathbf{I}_{2}
\end{array}\right], i=1,2,3, \quad D_{4}(j)=\left[\begin{array}{c}
0, \\
0.1
\end{array}\right], j=1,2, \\
& \Sigma_{i}(j)=\left[\begin{array}{ll}
0, & 1
\end{array}\right], \quad i=1,2,3, \quad \Sigma_{4}(j)=1, j=1,2, \\
& \Gamma_{i}(j)=\left[\begin{array}{ll}
0, & 0.0910
\end{array}\right], i=1,2,3,4, j=1,2,
\end{aligned}
$$


Note that in the uncertain system model presented in Pota et al. (2006), the uncertainty is introduced to account for higher order terms arising due to linearization of the underlying nonlinear system about its operating points. This type of uncertainty falls into the class of norm bounded uncertainties since the right-hand sides of the underlying nonlinear differential equations are sufficiently smooth in the vicinity of operating points. Therefore in the corresponding IQCs, the matrices $M_{i}, \hat{M}_{i}$ can be chosen to be arbitrary positive definite matrices; see Example 10. In this example, we selected $M_{i}=\hat{M}_{i}=I_{2 \times 2}, x_{i 0}=\left[\begin{array}{ll}0.1 & 0.1\end{array}\right]^{\prime}$, $i=1,2,3, M_{4}=\hat{M}_{4}=1, x_{40}=0.1414$ in the constraints (3) and (4). This selection provides for additional robustness against possible noises in the interconnections and mismatches in the operating point settings.

In addition to the rank constrained LMI approach detailed in the paper, we also tried using the Matlab function fmincon to find the upper bound in (15). This was unsuccessful since, despite numerous trials, a feasible initial estimate could not be found. (In this example, the system comprises only 4 subsystems. For a large-scale system, finding a feasible initial point for fmincon would be even harder.) Using the LMIRank algorithm (Orsi, 2005) applied to the optimization problem (48), the solution found was $\tau_{1}^{*}=$ $0.0338, \tau_{2}^{*}=0.0447, \tau_{3}^{*}=0.0367, \tau_{4}^{*}=0.0005, \theta_{1}^{*}=0.0163, \theta_{2}^{*}=0.0215, \theta_{3}^{*}=0.0105, \theta_{4}^{*}=0.0001$, resulting in an upper bound of 0.03155 . For the purpose of comparison, we also solved the optimization problem (50) arising in the corresponding state feedback problem. For that problem, solving (50) gives the optimal minimax value of the performance cost. In our simulation this optimal minimax value was computed to be equal to 0.03153 , which shows that our results achieved in the output feedback case are pretty good. The parameters of the corresponding dynamic output feedback controllers (12) are also given in the Appendix.

\section{Conclusion}

This paper considers a decentralized robust control design problem for an interconnected system subject to uncertain disturbances and randomly changing parameters. It extends the state feedback results of Ugrinovskii \& Pota (2005) to the output feedback case. A necessary and sufficient condition for the existence of an absolutely stabilizing decentralized switching controller is obtained. This condition involves a parameterized set of mode-dependent coupled algebraic Riccati equations and inequalities. Unlike Ugrinovskii \& Pota (2005), it is currently not clear whether an optimal LTI controller exists for the corresponding minimax optimal robust performance problem. However, a suboptimal controller and a corresponding upper bound on its robust performance are obtained and it is shown that this bound can be optimized by solving an equivalent optimization problem involving rank constrained LMIs. Using the LMIRank software, a numerical approach for solving this problem is presented. This approach performs well in the described example application, and our experience with other examples has been similar.

\section{Acknowledgements}

We thank Dr. H. R. Pota and Mr. G. X. Athanasius for their assistance in providing the example. We thank the Associate Editor and the reviewers for their comments.

\section{References}

Ait Rami, M., \& El Ghaoui, L. (1996). LMI optimization for nonstandard Riccati equations arising in stochastic control. IEEE Trans. on Autom. Contr., 41(11):1666-1671.

Başar, T., \& Bernhard, P. (1995). $H^{\infty}$-optimal control and related minimax design problems: a dynamic game approach (2nd ed.). Birkhäuser, Boston. 
Caines, P., \& Chen, H. (1985). Optimal adaptive LQG control for systems with finite state process parameters. IEEE Trans. on Autom. Contr., 30(2):185-189.

Davis, M. H. A. (1992). Markov models and optimization. Monographs on statistics and applied probability. Chapman and Hall, London.

de Farias, D. P., Geromel, J. C., do Val, J. B. R., \& Costa, O. L. V. (2000). Output feedback control of Markov jump linear systems in continuous-time. IEEE Trans. on Autom. Contr., 45(5):944-949.

de Souza, C. E., \& Fragoso, M. D. (1993). $H^{\infty}$ control for linear-systems with Markovian jumping parameters. Control-Theory and Advanced Technology, 9(2):457-466.

Doob, J. L. (1953). Stochastic processes. Wiley, New York.

Dullerud, G. E., \& Paganini, F. (2000). A course in robust control theory : a convex approach. Springer, New York.

El Ghaoui, L., \& Gahinet, P. (1993). Rank minimization under LMI constraints: a framework for output feedback problems. In Proc. 2nd ECC, Gröningen, The Netherlands (pp. 1176-1179).

Feng, X., Loparo, K. A., Ji, Y., \& Chizeck, H. J. (1992). Stochastic stability properties of jump linear systems. IEEE Trans. on Autom. Contr., 37(1):38-53.

Griffiths, B. E., \& Loparo, K. A. (1985). Optimal control of jump-linear Gaussian systems. Int. J. Contr., 42(4):791-820.

Ji, Y., \& Chizeck, H. J. (1990). Controllability, stabilizability, and continuous-time Markovian jump linear quadratic control. IEEE Trans. on Autom. Contr., 35(7):777-788.

Ji, Y., \& Chizeck, H. J. (1992). Jump linear quadratic Gaussian control in continuous time. IEEE Trans. on Autom. Contr., 37(12):1884-1892.

Li, L., \& Ugrinovskii, V. A. (2006). On necessary and sufficient conditions for $H_{\infty}$ output feedback control of Markov jump linear systems. In Proc. 2006 CDC, San Diago, CA (pp. 5525-5530). Also to appear in IEEE Transactions on Automatic Control, 52(7), 2007, 1287-1292.

Mariton, M. (1990). Jump linear systems in automatic control. Marcel Dekker Inc., New York.

Mariton, M., \& Bertrand, P. (1985). Robust jump linear quadratic control: a mode stabilizing solution. IEEE Trans. on Autom. Contr., 30(11):1145-1147.

Orsi, R. (2005). LMIRank: software for rank constrained LMI problems. Available from http://rsise.anu.edu.au/ robert/lmirank/.

Orsi, R., Helmke, U., \& Moore, J. B. (2006). A Newton-like method for solving rank constrained linear matrix inequalities. Automatica, 42(11):1875-1882.

Pakshin, P. V. (2003). Robust decentralized control of systems of random structure. Int. J. Computer and Systems Sciences, 42:200-204.

Pan, Z., \& Başar, T. (1995). $H_{\infty}$ control of Markovian jump systems and solutions to associated piecewisedeterministic differential games. In New Trends in Dynamic Games and Applications (G. J. Olsder, ed.), Birkhauser, Boston (pp. 61-94).

Petersen, I. R., \& McFarlane, D. C. (1994). Optimal guaranteed cost control and filtering for uncertain linear systems. IEEE Trans. on Autom. Contr., 39(9):1971-1977. 
Petersen, I. R., Ugrinovskii, V. A., \& Savkin, A. V. (2000). Robust control design using $H_{\infty}$ methods. Springer, London.

Pota, H. R., Athanasius, G. X., Ugrinovskii, V. A., \& Li, L. (2006). Control design for interconnected power systems with OLTCs via robust decentralized control. In Proc. 2006 ACC, Minneapolis, MN (pp. 3469-3474).

Qiu, W., Vittal, V., \& Khammash, M. (2004). Decentralized power system stabilizer design using linear parameter varying approach. IEEE Trans. on Power Systems, 19(4):1951-1960.

Ramos, R. A., Li, L., Ugrinovskii, V. A., \& Pota, H. R. (2006). Design of switching damping controllers for power systems based on a Markov jump parameter system approach. In Proc. 2006 CDC, San Diago, CA (pp. 4014-4019).

Sandell, N. R., Varaya, P., Athans, M., \& Safonov, M. (1978). Survey of decentralized control methods for large scale systems. IEEE Trans. on Autom. Contr., AC-23:108-128.

Savkin, A. V., \& Petersen, I. R. (1995). Minimax optimal control of uncertain systems with structured uncertainty. Int. J. Robust and Nonlinear Control, 5(2):119-137.

Siljak, D. D. (1991). Decentralized control of complex systems. Academic Press.

Ugrinovskii, V. A., Petersen, I. R., Savkin, A. V., \& Ugrinovskaya, E. Y. (2000). Decentralized statefeedback stabilization and robust control of uncertain large-scale systems with integrally constrained interconnections. Systems \& Control Letters, 40(2):107-119.

Ugrinovskii, V. A., \& Pota, H. R. (2005). Decentralized control of power systems via robust control of uncertain Markov jump parameter systems. Int. J. Contr., 78(9):662-677.

Wang, Y., Xie, L., \& de Souza, C. E. (1995). Robust decentralized control of interconnected uncertain linear systems. In Proc. 34th CDC, Los Angeles, CA (pp. 2653-8).

Willsky, B. C., \& Levy, A. S. (1979). Stochastic stability research for complex power systems. Technical report, Mass. Inst. Technology, Cambridge.

Yakubovich, V. A. (1988). Dichotomy and absolute stability of nonlinear systems with periodically nonstationary linear part. Systems \& Control Letters, 11(3):221-228. 


\section{Appendix}

The power system discussed in the example is shown in Fig. 2. In Pota et al. (2006), linear models are obtained for two different load profiles (from Bus 4 to Bus 9) as indicated in the figure. Parameters of the corresponding linearized subsystems corresponding to the two load profiles under consideration are as follows:

$$
\begin{aligned}
& A_{1}(1)=\left[\begin{array}{cc}
0 & 1 \\
-0.614 & -0.1235
\end{array}\right] \text {, } \\
& A_{1}(2)=\left[\begin{array}{cc}
0 & 1 \\
-0.4955 & -0.1235
\end{array}\right] \text {, } \\
& A_{2}(1)=\left[\begin{array}{cc}
0 & 1 \\
-0.4540 & -0.0962
\end{array}\right] \text {, } \\
& A_{2}(2)=\left[\begin{array}{cc}
0 & 1 \\
-0.3928 & -0.0962
\end{array}\right] \text {, } \\
& A_{3}(1)=\left[\begin{array}{cc}
0 & 1 \\
-0.6096 & -0.1164
\end{array}\right] \text {, } \\
& A_{3}(2)=\left[\begin{array}{cc}
0 & 1 \\
-0.5366 & -0.1164
\end{array}\right] \text {, } \\
& A_{4}(1)=-0.9780 \text {, } \\
& B_{1}(1)=\left[\begin{array}{cc}
0 & 0 \\
0.1235 & -0.0701
\end{array}\right] \text {, } \\
& A_{4}(2)=-0.8842 \text {, } \\
& B_{2}(1)=\left[\begin{array}{cc}
0 & 0 \\
0.0962 & -0.0730
\end{array}\right] \text {, } \\
& B_{1}(2)=\left[\begin{array}{cc}
0 & 0 \\
0.1235 & -0.0983
\end{array}\right] \text {, } \\
& B_{3}(1)=\left[\begin{array}{cc}
0 & 0 \\
0.1164 & -0.0604
\end{array}\right] \text {, } \\
& B_{2}(2)=\left[\begin{array}{cc}
0 & 0 \\
0.0962 & -0.0505
\end{array}\right] \text {, } \\
& B_{4}(1)=10 \text {, } \\
& B_{3}(2)=\left[\begin{array}{cc}
0 & 0 \\
0.1164 & -0.0410
\end{array}\right] \text {, } \\
& E_{i}(j)=\left[\begin{array}{ll}
0.1 & 0 \\
0.1 & 0
\end{array}\right], i=1,2,3, \\
& B_{4}(2)=10 \text {, } \\
& H_{i}(j)=\left[\begin{array}{ll}
1 & 0 \\
0 & 0
\end{array}\right], i=1,2,3, \\
& E_{4}(j)=\left[\begin{array}{ll}
0.1 & 0
\end{array}\right], j=1,2, \\
& G_{i}(j)=\left[\begin{array}{ll}
0 & 0 \\
0 & 1
\end{array}\right], i=1,2,3, \\
& H_{4}(j)=\left[\begin{array}{l}
1 \\
0
\end{array}\right], j=1,2, \\
& G_{4}(j)=\left[\begin{array}{l}
0 \\
1
\end{array}\right], j=1,2, \\
& L_{1}(1)=\left[\begin{array}{cccccccccc}
0 & 0 & 0 & 0 & 0 & 0 & 0 & 0 & 0 & 0 \\
0.0028 & 0 & 0.0067 & 0 & 0.0140 & 0 & -0.0635 & 0 & -0.0647 & 0
\end{array}\right] \text {, } \\
& L_{1}(2)=\left[\begin{array}{cccccccccc}
0 & 0 & 0 & 0 & 0 & 0 & 0 & 0 & 0 & 0 \\
0.0026 & 0 & 0.0060 & 0 & 0.0336 & 0 & -0.0744 & 0 & -0.0803 & 0
\end{array}\right] \text {, } \\
& L_{2}(1)=\left[\begin{array}{cccccccccc}
0 & 0 & 0 & 0 & 0 & 0 & 0 & 0 & 0 & 0 \\
0.0022 & 0 & 0.0097 & 0 & 0.0146 & 0 & -0.0675 & 0 & -0.0679 & 0
\end{array}\right] \text {, } \\
& L_{2}(2)=\left[\begin{array}{cccccccccc}
0 & 0 & 0 & 0 & 0 & 0 & 0 & 0 & 0 & 0 \\
0.0022 & 0 & 0.0094 & 0 & 0.0159 & 0 & -0.0256 & 0 & -0.0372 & 0
\end{array}\right] \text {, } \\
& L_{3}(1)=\left[\begin{array}{cccccccccc}
0 & 0 & 0 & 0 & 0 & 0 & 0 & 0 & 0 & 0 \\
0.0063 & 0 & 0.0118 & 0 & 0.0121 & 0 & -0.0556 & 0 & -0.0548 & 0
\end{array}\right] \text {, } \\
& L_{3}(2)=\left[\begin{array}{cccccccccc}
0 & 0 & 0 & 0 & 0 & 0 & 0 & 0 & 0 & 0 \\
0.0062 & 0 & 0.0118 & 0 & 0.0133 & 0 & -0.0168 & 0 & -0.0252 & 0
\end{array}\right] \text {, } \\
& L_{4}(1)=\left[\begin{array}{llllllllllll}
0 & 0 & 0 & 0 & 0 & 0 & -0.1168 & 0 & -0.1166 & 0 & -0.1168 & 0
\end{array}\right] \text {, } \\
& L_{4}(2)=\left[\begin{array}{llllllllllll}
0 & 0 & 0 & 0 & 0 & 0 & -0.0156 & 0 & -0.0174 & 0 & -0.0179 & 0
\end{array}\right] \text {. }
\end{aligned}
$$




\section{MACHINE 9 BUS LAYOUT}

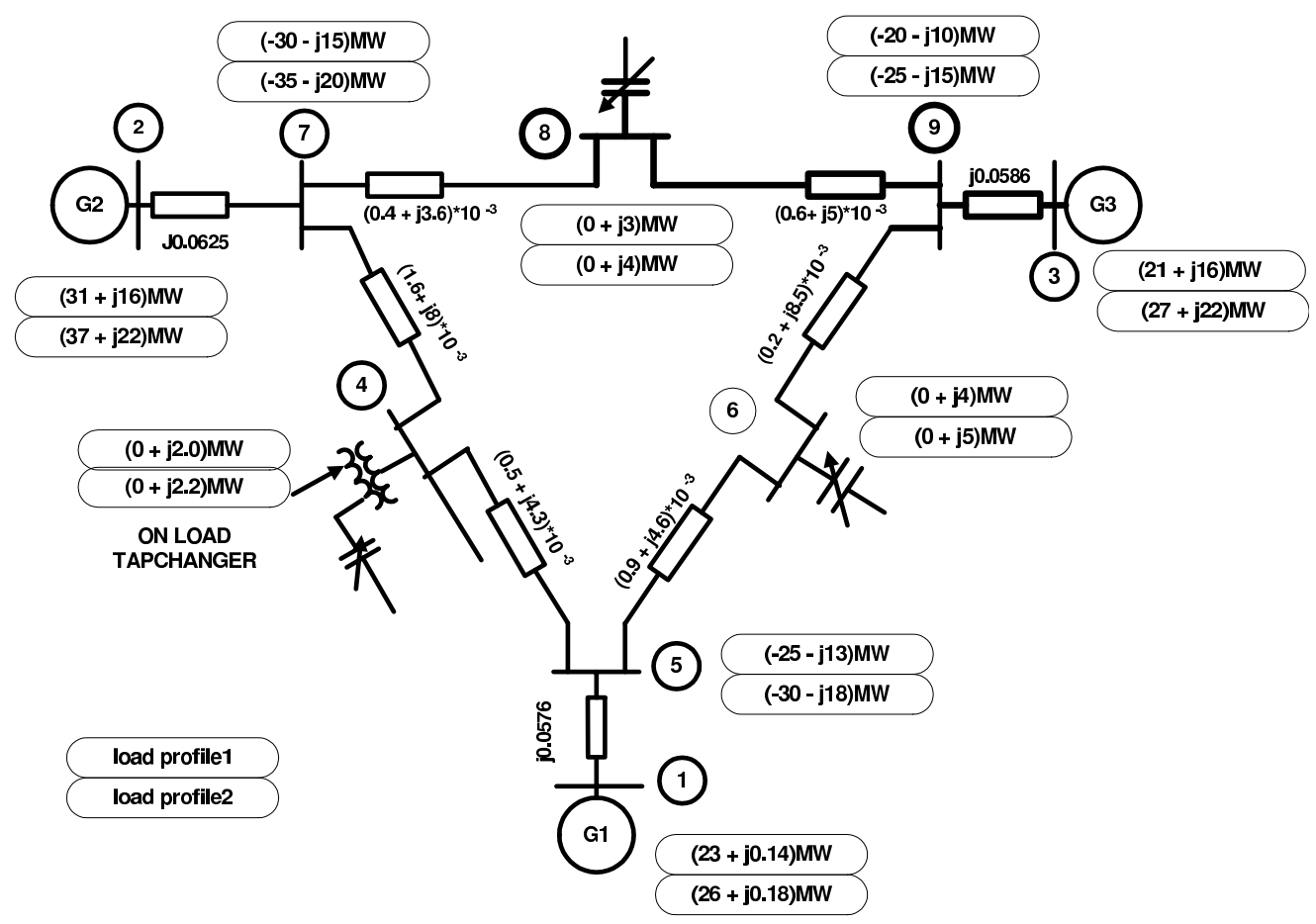

Figure 2: 9-Bus power system of Pota et al. (2006).

The corresponding parameters of the controller were obtained as follows:

$$
\begin{array}{rlrl}
A_{c, 1}(1) & =\left[\begin{array}{cc}
0.0879 & 7.2915 \\
-0.6094 & -5.3086
\end{array}\right], & & A_{c, 1}(2)=\left[\begin{array}{cc}
0.0988 & 13.8819 \\
-0.4822 & -6.8623
\end{array}\right], \\
A_{c, 2}(1) & =\left[\begin{array}{cc}
0.0957 & 10.4503 \\
-0.4271 & -5.5272
\end{array}\right], & A_{c, 2}(2) & =\left[\begin{array}{cc}
0.0798 & 7.5840 \\
-0.4183 & -4.3519
\end{array}\right], \\
A_{c, 3}(1) & =\left[\begin{array}{cc}
0.1006 & 7.2718 \\
-0.5827 & -5.2063
\end{array}\right], & & A_{c, 3}(2)=\left[\begin{array}{cc}
0.0834 & 5.3587 \\
-0.5699 & -3.9422
\end{array}\right], \\
A_{c, 4}(1) & =-433.0048, & & A_{c, 4}(2)=-11.8305, \\
B_{c, 1}(1) & =\left[\begin{array}{cc}
-6.1632 \\
4.9336
\end{array}\right], & B_{c, 1}(2) & =\left[\begin{array}{c}
-12.7109 \\
6.5070
\end{array}\right], \\
B_{c, 2}(1) & =\left[\begin{array}{cc}
-9.2638 \\
5.2266
\end{array}\right], & & B_{c, 2}(2)=\left[\begin{array}{cc}
-6.4330 \\
3.9408
\end{array}\right], \\
B_{c, 3}(1) & =\left[\begin{array}{cc}
-6.1181 \\
4.8740
\end{array}\right], & & B_{c, 3}(2)=\left[\begin{array}{cc}
-4.2330 \\
3.4904
\end{array}\right], \\
B_{c, 4}(1) & =423.9681, & & B_{c, 4}(2)=1.8699, \\
K_{c, 1}(1) & =\left[\begin{array}{cc}
-0.9619 & -4.3842 \\
0.0719 & 0.3277
\end{array}\right], & K_{c, 1}(2) & =\left[\begin{array}{cc}
-1.2471 & -5.8821 \\
0.1308 & 0.6171
\end{array}\right], \\
K_{c, 2}(1) & =\left[\begin{array}{cc}
-1.2000 & -6.8159 \\
0.1116 & 0.6338
\end{array}\right], & K_{c, 2}(2)=\left[\begin{array}{cc}
-1.1967 & -5.2938 \\
0.0769 & 0.3400
\end{array}\right], \\
K_{c, 3}(1) & =\left[\begin{array}{cc}
-1.0907 & -5.4676 \\
0.0669 & 0.3354
\end{array}\right], & K_{c, 3}(2)=\left[\begin{array}{cc}
-1.0837 & -4.2799 \\
0.0451 & 0.1780
\end{array}\right], \\
K_{c, 4}(1)=-0.9985, & & K_{c, 4}(2)=-0.9222 .
\end{array}
$$

